\title{
FUNCTIONAL CALCULUS FOR GENERATORS OF SYMMETRIC CONTRACTION SEMIGROUPS
}

\author{
ANDREA CARBONARO AND OLIVER DRAGIČEVIĆ
}

\begin{abstract}
We prove that every generator of a symmetric contraction semigroup on a $\sigma$-finite measure space admits, for $1<p<\infty$, a Hörmander-type holomorphic functional calculus on $L^{p}$ in the sector of angle $\phi_{p}^{*}=\arcsin |1-2 / p|$. The obtained angle is optimal.
\end{abstract}

\section{INTRODUCTION}

Suppose that $(\Omega, \nu)$ is a $\sigma$-finite measure space. Let $\mathcal{A}$ be a nonnegative, possibly unbounded, self-adjoint operator on $L^{2}(\Omega, \nu)$, and let $\left\{\mathcal{P}_{\lambda}\right\}$ be the spectral resolution of the identity for which

$$
\mathcal{A} f=\int_{0}^{\infty} \lambda \mathrm{d} \mathcal{P}_{\lambda} f, \quad f \in \mathrm{D}(\mathcal{A}) .
$$

If $m$ is a bounded Borel function on $[0, \infty)$ then, by the spectral theorem, the operator

$$
m(\mathcal{A}) f=\int_{0}^{\infty} m(\lambda) \mathrm{d} \mathcal{P}_{\lambda} f, \quad f \in L^{2}(\Omega, \nu)
$$

is bounded on $L^{2}(\Omega, \nu)$. If $m(\mathcal{A})$ extends to a bounded operator on $L^{p}(\Omega, \nu)$ for some $p \in[1, \infty]$, we say that $m$ is a $L^{p}$ spectral multiplier for $\mathcal{A}$. We denote by $\mathcal{M}_{p}(\mathcal{A})$ the class of all $L^{p}$ spectral multipliers for $\mathcal{A}$.

We shall always assume that $\mathcal{A}$ is the generator of a symmetric contraction semigroup, i.e., we always assume that the semigroup $(\exp (-t \mathcal{A}))_{t>0}$ satisfies the contraction property:

$$
\|\exp (-t \mathcal{A}) f\|_{p} \leqslant\|f\|_{p}, \quad f \in L^{2}(\Omega, \nu) \cap L^{p}(\Omega, \nu),
$$

whenever $t>0$ and $1 \leqslant p \leqslant \infty$.

An interesting and challenging problem consists in characterizing the class $\mathcal{M}_{p}(\mathcal{A})$ when $1<p<\infty$. This question is still open, even for the Euclidean Laplacian [24, 26]. It is well known that $\mathcal{M}_{p}(\mathcal{A})$ depends on the generator $\mathcal{A}$, the measure space $(\Omega, \nu)$ and the index $p$ [36, 37, 27, 12, 25, 15.

In this paper we pursue the study of "universal" multiplier theorems; namely, for every $p \in(1, \infty)$ we are looking for conditions on $m$ which imply that $m \in \mathcal{M}_{p}(\mathcal{A})$ for all generators $\mathcal{A}$ of symmetric contraction semigroups. The first universal multiplier theorem was proved by E. M. Stein, who showed that if $m$ is of Laplace transform type then $m \in \mathcal{M}_{p}(\mathcal{A})$ for all generators $\mathcal{A}$ of Markovian semigroups and every $p \in(1, \infty)$ [47. Corollary 3, p. 121]. Conditions on $m$ which imply that $m \in \mathcal{M}_{p}(\mathcal{A})$ are often expressed in terms of "regularity" of the multiplier.

For every $\phi \in(0, \pi / 2]$ consider the sector

$$
\mathbf{S}_{\phi}=\{z \in \mathbb{C} \backslash\{0\}:|\arg z|<\phi\},
$$

and denote by $H^{\infty}\left(\mathbf{S}_{\phi}\right)$ the algebra of all bounded holomorphic functions on $\mathbf{S}_{\phi}$. By Fatou's theorem, every $m \in H^{\infty}\left(\mathbf{S}_{\phi}\right)$ admits a nontangential limit almost everywhere 
on the boundary of $\mathbf{S}_{\phi}$. By a slight abuse of notation, if $m \in H^{\infty}\left(\mathbf{S}_{\phi}\right)$ we use the same symbol $m$ to denote its extension to the closure $\overline{\mathbf{S}}_{\phi}$. We assume that $m$ is defined at zero, so that we will be able to consider noninjective generators.

For every $J \in[0, \infty)$ we denote by $H^{\infty}\left(\mathbf{S}_{\phi} ; J\right)$ the class of all functions in $H^{\infty}\left(\mathbf{S}_{\phi}\right)$ which satisfy the Mihlin-Hörmander condition of order $J$ on the boundary of $\mathbf{S}_{\phi}$. Explicitly, fix a smooth function $\psi: \mathbb{R} \rightarrow[0,1]$ supported in $(1 / 4,4)$ and such that $\psi=1$ on $[1 / 2,2]$. Let $H^{J}(\mathbb{R})$ denote the usual $L^{2}$-Sobolev space of order $J$. Then

$$
H^{\infty}\left(\mathbf{S}_{\phi} ; J\right)=\left\{m \in H^{\infty}\left(\mathbf{S}_{\phi}\right):\|m\|_{\phi ; J}<\infty\right\},
$$

where

$$
\|m\|_{\phi ; J}=\sup _{R>0}\left\|\psi m\left(e^{i \phi} R \cdot\right)\right\|_{H^{J}(\mathbb{R})}+\sup _{R>0}\left\|\psi m\left(e^{-i \phi} R \cdot\right)\right\|_{H^{J}(\mathbb{R})} .
$$

It is known that $H^{\infty}\left(\mathbf{S}_{\phi} ; J\right)$ endowed with the norm $\|\cdot\|_{\phi ; J}$ is a Banach space and that $H^{\infty}\left(\mathbf{S}_{\phi}\right) \subset H^{\infty}\left(\mathbf{S}_{\psi} ; J\right)$ as Banach spaces, for all $\phi>\psi$ and $J \geqslant 0$.

It has been known for a while that generators of symmetric contraction semigroups have a bounded holomorphic functional calculus on $L^{p}$ in sectors of angle smaller than $\pi / 2$. More precisely, for every $p \in(1, \infty)$ there exists an angle $\vartheta_{p} \in(0, \pi / 2)$ such that if $\vartheta>\vartheta_{p}$ and $m \in H^{\infty}\left(\mathbf{S}_{\vartheta}\right)$, then $m \in \mathcal{M}_{p}(\mathcal{A})$ and there exists $C(p, \vartheta)>0$ such that

$$
\|m(\mathcal{A})\|_{p} \leqslant C(p, \vartheta)\left(\|m\|_{\vartheta ; 0}+|m(0)|\right) .
$$

Indeed, M. Cowling [13] improved Stein's universal multiplier theorem by showing that

$$
\vartheta_{p}=\phi_{p}^{C}=\pi\left|\frac{1}{p}-\frac{1}{2}\right|
$$

suffices for all generators of symmetric contraction semigroups. In the special case when $\mathcal{A}$ generates a sub-Markovian semigroup, Cowling's result has been improved by P. C. Kunstmann and Ž. Štrkalj [31] who showed that $\phi_{p}^{C}$ can be replaced by

$$
\phi_{p}^{K \breve{S}}=\frac{\pi}{2}\left|\frac{1}{p}-\frac{1}{2}\right|+\left(1-\left|\frac{1}{p}-\frac{1}{2}\right|\right) \arcsin \frac{|p-2|}{2 p-|p-2|} .
$$

Recently C. Kriegler [30, Remark 2] extended this result to generators of symmetric contraction semigroups.

For every $p \in(1, \infty)$ define

$$
\phi_{p}^{*}=\arcsin \left|1-\frac{2}{p}\right| .
$$

Note that $0<\phi_{p}^{*}<\phi_{p}^{K \breve{S}}<\phi_{p}^{C}$ whenever $p \neq 2$. Interestingly, $\phi_{p}^{*}$ relates to the angle of analyticity on $L^{p}$ of all symmetric contraction semigroups. Indeed, as a consequence of a more general result by Kriegler [30, Theorem 5.4 and Corollary 6.2], any symmetric contraction semigroup is contractive and holomorphic on $L^{p}$ in the sector $\mathbf{S}_{\pi / 2-\phi_{p}^{*}}$ (see Proposition 3).

It is known that for some specific generators the angle $\phi_{p}^{*}$ suffices. For example, Laplacians on Euclidean spaces and, more generally, Laplace-Beltrami operators on doubling Riemannian manifolds whose heat kernels satisfy gaussian estimates admit a Hörmander-type functional calculus [27, [21, Theorem 3.1], [42, Theorem 7.23]. Consequently these operators have a bounded holomorphic functional calculus on $L^{p}$ in any sector. It is also known that $\phi_{p}^{*}$ suffices for Laplace-Beltrami operators on symmetric spaces of noncompact type [12, 46, 2, 1] and, more generally, on manifolds with bounded geometry and optimal $L^{2}$ spectral gap [51, 34, 52. However, all of the results above 
entail estimates of the $L^{p}$ norms of the multipliers which depend on the dimension of the underlying space, while universal multiplier theorems give dimension-free estimates as in (11).

The given examples involve Riemannian manifolds where the volume of balls satisfies the doubling property at least for small radii. The problem of reducing the angle $\phi_{p}^{K \breve{S}}$ for Laplacians on (weighted) manifolds that are not locally doubling seems to be more difficult to solve, even with dimension-dependent estimates. Indeed, to the best of our knowledge, the only example studied and well understood in this framework is the symmetric finite-dimensional Ornstein-Uhlenbeck operator, i.e., the weighted Laplacian

$$
\mathcal{L}_{O U}=\Delta_{\mathbb{R}^{n}}+x \cdot \nabla_{\mathbb{R}^{n}}
$$

on the Euclidean space $\mathbb{R}^{n}$ endowed with the Gaussian measure

$$
\mathrm{d} \gamma_{n}(x)=(2 \pi)^{-n / 2} e^{-|x|^{2} / 2} \mathrm{~d} x .
$$

In [23, Theorem 1] and [33, Theorem 1.2] it was proved that $\phi_{p}^{*}$ suffices for $\mathcal{L}_{O U}$. This was extended in [45] to the Laguerre operators and in 9] to certain perturbations of $\mathcal{L}_{O U}$. The proofs in [23, 33, rely heavily on properties of the particular operator in question. In particular, the authors use an explicit formula for the heat kernel with complex time for $\mathcal{L}_{O U}$ (Mehler formula) and, combining it with a change of variable in time and a stationary phase argument, they prove that for every $p>1$ there exists $C(n, p)>0$ such that

$$
\left\|\mathcal{L}_{O U}^{i s}\right\|_{\mathcal{B}\left(L^{p}\left(\mathbb{R}^{n}, \gamma_{n}\right)\right)} \leqslant C(n, p) e^{\phi_{p}^{*}|s|}
$$

for all $s \in \mathbb{R}$. By a result of Meda (see Propositions 5 and 7 ), the estimate above implies that for every $J>1$ there exists $\widetilde{C}(n, p, J)>0$ such that if $m \in H^{\infty}\left(\mathbf{S}_{\phi_{p}^{*}} ; J\right)$, then

$$
\left\|m\left(\mathcal{L}_{O U}\right)\right\|_{\mathcal{B}\left(L^{p}\left(\mathbb{R}^{n}, \gamma_{n}\right)\right)} \leqslant \widetilde{C}(n, p, J)\left(\|m\|_{\phi_{p}^{*} ; J}+|m(0)|\right) .
$$

The estimates in (3) and (4) depend on the dimension of the underlying space, $\mathbb{R}^{n}$. This is rather unpleasant, since it does not provide any information on the functional calculus for infinite-dimensional Ornstein-Uhlenbeck operators, i.e., Ornstein-Uhlenbeck operators on Wiener spaces.

Main result. Few years ago, G. Mauceri and S. Meda remarked to the first named author of the present paper that $\phi_{p}^{*}$ could be the optimal angle in the universal multiplier theorem for generators of symmetric contraction semigroups. The aim of this paper is to confirm this supposition by showing that, for every generator of a symmetric contraction semigroup, the angle $\phi_{p}^{K \breve{S}}$ can be replaced by $\phi_{p}^{*}$. Namely, we prove the following result. First let us introduce the notation $p^{*}=\max \{p, p /(p-1)\}$ for $p>1$.

Theorem 1 (Multiplier theorem). For every generator $\mathcal{A}$ of a symmetric contraction semigroup on a $\sigma$-finite measure space $(\Omega, \nu)$, every $p \in(1, \infty), J>3 / 2$ and $m \in$ $H^{\infty}\left(\mathbf{S}_{\phi_{p}^{*}} ; J\right)$, the operator $m(\mathcal{A})$ extends to a bounded operator on $L^{p}(\Omega, \nu)$, and there exists $C(J)>0$ such that

$$
\|m(\mathcal{A})\|_{\mathcal{B}\left(L^{p}(\Omega, \nu)\right)} \leqslant C(J)\left(p^{*}-1\right)^{9 / 4} \log p^{*}\left(\|m\|_{\phi_{p}^{*} ; J}+|m(0)|\right) .
$$

In short, generators of symmetric contraction semigroups admit a Hörmander-type holomorphic functional calculus of order $J>3 / 2$ in the sector $\mathbf{S}_{\phi_{p}^{*}}$. We remark that, independently of the order of the Hörmander condition, the angle $\phi_{p}^{*}$ in Theorem 1 is sharp, since it is optimal for the Ornstein-Uhlenbeck operator $\mathcal{L}_{O U}$; see [23, Theorem $2]$ and [25, Theorem 2.2]. 
Consequences of the multiplier theorem. Apart from improving the aforementioned results of Stein [47, Cowling [13] and Kunstmann and Strkalj [31], our multiplier theorem in particular applies to the (finite as well as infinite-dimensional) OrnsteinUhlenbeck operator and, because we get dimension-free estimates, it improves multiplier theorems in [23, 33]. However, the result in [33] requires a weaker order of differentiability of the multiplier, namely $J>1$.

By combining Theorem 1 with the techniques developed by Cowling in [13, Section $3]$ we improve his maximal theorems [13, Theorems 7 and 8]. The precise formulation of this statement is contained in Theorem 12. This theorem, together with a standard argument of Stein [47, pp. 72-82], in turn leads to new results concerning pointwise convergence $\exp (-z \mathcal{A}) f \rightarrow f$ as $z \rightarrow 0$ in the closed sector $\overline{\mathbf{S}}_{\phi}$, for $0 \leqslant \phi<\phi_{p}$; see Corollary 13 for a concise statement.

By Theorem 1 and [14, Corollary 6.7] we obtain the following square-function estimates. Let $\phi>\phi_{p}^{*}$ and $v, C>0$. Suppose that $\psi \in H^{\infty}\left(\mathbf{S}_{\phi}\right)$ satisfies $|\psi(\zeta)| \leqslant$ $C|\zeta|^{v} /\left(1+|\zeta|^{2}\right)^{v}$ for all $\zeta \in \mathbf{S}_{\phi}$. Then

$$
\left\|\left(\int_{0}^{\infty}|\psi(t \mathcal{A}) f(\cdot)|^{2} \frac{\mathrm{d} t}{t}\right)^{1 / 2}\right\|_{p} \leqslant C(p, \phi)\|f\|_{p}, \quad f \in L^{p}(\Omega, \nu) .
$$

We also mention that Theorem 1 combined with [28, Theorem 6.5] implies that, for $1<p<\infty$ and $0<\alpha<\pi /\left(2 \phi_{p}^{*}\right)$, the operator $\mathcal{A}_{p}^{\alpha}$ has the so-called maximal $L^{q}$-regularity for all $1<q<\infty$; see, for example, [28] and [31, Section 4]. Moreover, by combining Theorem 1 with [28, Theorem 5.3], we deduce that $\mathcal{A}$ is $R$-sectorial on $L^{p}(\Omega, \nu)$ of angle $\phi_{p}^{*}$. We refer the reader to [28] and the references therein for a detailed discussion about Rademacher boundedness and some of its consequences.

Outline of the proof. Our approach consists of proving suitable $L^{p}$ estimates of imaginary powers of $\mathcal{A}$ (Proposition 11) which will then, through a theorem of Meda (Propositions 5 and 17), imply the multiplier theorem. In order to prove the estimates of the imaginary powers we first derive a bilinear embedding theorem with complex time (Theorem 9).

Theorem 9 is proven by studying monotonicity properties of the heat flow associated with a particular Bellman function defined by Nazarov and Treil [38. This reduces the problem to proving new convexity properties of the Bellman function in question (Theorem 15).

The proof of Theorem 9 can be viewed, on one hand, as an extension of the Bellman function technique, widely known since the mid 1990s when it was introduced in harmonic analysis by Nazarov, Treil and Volberg in the first preprint version of their paper [39] and afterwards employed in a large number of papers, of which the closest ones to our approach are [43, 41, 18, 19, 20, 10. On the other hand, it may be considered as an extension of the heat flow method used by Bakry 3 for proving analyticity properties of symmetric diffusion semigroups. When studying the heat flow associated with the Bellman function, we put emphasis on encompassing complex times and obtaining, apart from the monotonicity, also quantitative estimates of the flow's derivative.

Let us, for the reader's convenience, put down a scheme summarizing the mutual dependence of the results in this paper:

$$
\left.\left.\begin{array}{r}
\text { Bellman function properties } \\
\text { Heat flow }
\end{array}\right\} \Rightarrow \text { Bilinear embedding } \Rightarrow \begin{array}{r}
\text { Estimates of }\left\|\mathcal{A}^{i s}\right\|_{p} \\
\text { Theorem of Meda }
\end{array}\right\} \Rightarrow \text { Multiplier theorem }
$$


At the end let us mention that, for all we know, Bellman functions have so far never been used for the purpose of proving general spectral multiplier theorems. We plan to further extend this method, in particular to analogue problems on UMD spaces.

Organization of the paper. In Section 2 we introduce much of the notation and invoke some of the basic objects and known results that will be used in the rest of the paper, among them the theorem of Meda about imaginary powers. In Section 3 we formulate the bilinear embedding theorem (Theorem 9) and show that it, together with Meda's result, implies the multiplier theorem (Theorem 1). The rest of the paper is then devoted to establishing Theorem 9. The main idea utilized to that end is presented in Section 4. In Section 5 we define the Bellman function of Nazarov and Treil and formulate the crucial convexity property that this function satisfies (Theorem 15). This property is verified in Section [6. Finally, in Section 7 we complete the proof of the bilinear embedding and thus of the multiplier theorem.

\section{Preparation}

Given two quantities $A$ and $B$, we adopt the convention whereby $A \lesssim B$ means that there exists an absolute constant $C>0$ such that $A \leqslant C B$. If both $A \lesssim B$ and $B \lesssim A$, then we write $A \sim B$. If $\left\{\lambda_{1}, \ldots, \lambda_{n}\right\}$ is a set of parameters, $C\left(\lambda_{1}, \ldots, \lambda_{n}\right)$ denotes a constant depending only on $\lambda_{1}, \ldots, \lambda_{n}$. When $A \leqslant C\left(\lambda_{1}, \ldots, \lambda_{n}\right) B$, we will often write $A \lesssim \lambda_{1}, \ldots, \lambda_{n} B$.

If $B$ is a matrix-valued function on $\mathbb{R}^{N}$, more precisely, $B: \mathbb{R}^{N} \rightarrow \mathbb{R}^{M, M}$, then for $\xi \in \mathbb{R}^{N}$ and $\omega \in \mathbb{R}^{M}$ we set

$$
B[\xi ; \omega]=\langle B(\xi) \omega, \omega\rangle_{\mathbb{R}^{M}},
$$

where $\langle\cdot, \cdot\rangle_{\mathbb{R}^{M}}$ denotes the usual scalar product in $\mathbb{R}^{M}$.

Throughout the paper, the hypotheses on $(\Omega, \nu), \mathcal{A}$ and $\exp (-t \mathcal{A})$ will be as specified early in the introduction. In order to simplify the notation we set, for $\Re z>0$,

$$
T_{z}=\exp (-z \mathcal{A}) .
$$

The semigroup $\left(T_{t}\right)_{t>0}$ is strongly continuous for $1 \leqslant p<\infty$ and weak* continuous for $p=\infty$. When necessary to distinguish between different $p$, we denote by $\mathcal{A}_{p}$ the generator of $\left(T_{t}\right)_{t>0}$ on $L^{p}(\Omega, \nu)$. Furthermore, $\mathrm{D}\left(\mathcal{A}_{p}\right), \mathrm{R}\left(\mathcal{A}_{p}\right)$ and $\mathrm{N}\left(\mathcal{A}_{p}\right)$ will stand for the domain, range and null-space of $\mathcal{A}_{p}$, respectively. The following result is well known.

Lemma 2. The orthogonal projection $\mathcal{P}_{0}$ on $\mathrm{N}\left(\mathcal{A}_{2}\right)$ is given by

$$
\mathcal{P}_{0} f=\lim _{t \rightarrow \infty} T_{t} f, \quad f \in L^{2}(\Omega, \nu) .
$$

Moreover, for every $p \in(1, \infty)$ the following holds:

(i) the operator $\mathcal{P}_{0}$ extends to a contractive projection on $L^{p}(\Omega, \nu)$. In particular, $L^{p}(\Omega, \nu)=\overline{\mathrm{R}\left(\mathcal{A}_{p}\right)} \oplus \mathrm{N}\left(\mathcal{A}_{p}\right)$, where the sum is direct.

(ii) $\mathrm{D}\left(\mathcal{A}_{2}\right) \cap \mathrm{D}\left(\mathcal{A}_{p}\right)$ is dense both in $L^{2}(\Omega, \nu)$ and $L^{p}(\Omega, \nu)$, and $\mathrm{D}\left(\mathcal{A}_{2}\right) \cap \mathrm{R}\left(\mathcal{A}_{2}\right) \cap$ $L^{p}(\Omega, \nu)$ is dense in $\overline{\mathrm{R}\left(\mathcal{A}_{p}\right)}$.

For every $p \in[1, \infty]$, we denote the norm on $L^{p}(\Omega, \nu)$ by $\|\cdot\|_{p}$ and set

$$
q=p^{\prime}=p /(p-1) .
$$


By abuse of notation, if $S$ is a bounded operator on $L^{p}(\Omega, \nu)$, we denote its operator norm by $\|S\|_{p}$. If $\varphi \in L^{p}(\Omega, \nu)$ and $\psi \in L^{q}(\Omega, \nu)$, the dual pairing between $\phi$ and $\psi$ is denoted by

$$
\langle\varphi, \psi\rangle=\int_{\Omega} \varphi \bar{\psi} d \nu
$$

For any $\phi \in(-\pi / 2, \pi / 2)$, we define $\phi^{*}=\pi / 2-\phi$; with this notation, (2) implies

$$
\phi_{p}=\phi_{q}=\arccos \left|1-\frac{2}{p}\right|=\arctan \frac{2 \sqrt{p-1}}{|p-2|} .
$$

This is a small abuse of notation, since we previously denoted $p *=\max \{p, p /(p-1)\}$ for $p>1$. We however believe that the context will always make it clear whether the asterisk is being applied to a "Lebesgue exponent" or to an "angle".

Next result concerns analyticity on $L^{p}$ of symmetric contraction semigroups, and was first proven by Bakry [3, Thèoréme 3] for a certain class of sub-Markovian semigroups which contains, for example, those generated by weighted Laplace-Beltrami operators on complete manifolds. The result was than extended by Liskevich and Perelmuter 32 , Corollary 3.2] to all sub-Markovian semigroups; see also [42, Theorem 3.13]. Proposition 3 was recently obtained by Kriegler [30, Corollary 6.2], as a consequence of a more general result on noncommutative $L^{p}$ spaces. The above-mentioned results improve an earlier estimate of this kind due to Stein [47, III, §2, Theorem 1, page 67].

Proposition 3 (30). For every $p \in(1, \infty)$, the semigroup $\left(T_{t}\right)_{t>0}$ extends to a contractive analytic semigroup on $L^{p}(\Omega, \nu)$ in the sector $\mathbf{S}_{\phi_{p}}$.

Remark 4. By [47, p. 72], as a consequence of Proposition 3, if $p \in(1, \infty), \phi \in$ $\left(-\phi_{p}, \phi_{p}\right)$ and $f \in L^{p}(\Omega, \nu)$, then for almost every $x \in \Omega$ the function $t \mapsto\left(T_{t e^{i \phi}} f\right)(x)$ is real-analytic on $(0, \infty)$. This fact will be implicitly used in the rest of this paper.

If $M \in L^{\infty}\left(\mathbb{R}_{+}\right)$, define its Laplace transform $\widetilde{M}$ by the rule

$$
\widetilde{M}(\lambda)=\lambda \int_{0}^{\infty} M(t) e^{-t \lambda} \mathrm{d} t, \quad \Re \lambda>0 .
$$

A prominent special case of multipliers of Laplace transform type are the imaginary powers $\mathcal{A}^{i s}, s \in \mathbb{R}$. It is well known that there is a deep relation between the growth of the norms of imaginary powers of $\mathcal{A}$ and its holomorphic functional calculus on sectors [35, Theorem 4], [14, Theorem 5.4]. In particular, we have the following result, essentially due to Meda [35] and proved in [33, Theorem 2.1] (for the case $J \in \mathbb{N}$ we refer the reader to [23, Theorem 2.2]).

Proposition 5. Suppose that $1<p<\infty$ and that there exist constants $C_{0}, \sigma>0$ and $\phi^{*} \in[0, \pi / 2)$ such that

$$
\left\|\mathcal{A}^{i s} f\right\|_{p} \leqslant C_{0}(1+|s|)^{\sigma} e^{\phi^{*}|s|}\|f\|_{p}, \quad \forall f \in \mathrm{R}\left(\mathcal{A}_{p}\right), \quad \forall s \in \mathbb{R} .
$$

If $J>\sigma+1$ and $m \in H^{\infty}\left(\mathbf{S}_{\phi^{*}} ; J\right)$, then $m(\mathcal{A})$ extends to a bounded operator on $\overline{\mathrm{R}\left(\mathcal{A}_{p}\right)}$.

Since in Theorem 1 we shall also be interested in the behavior of the $L^{p}$ norm of the multiplier $m(\mathcal{A})$ with respect to $p$, we now briefly recall the proof of Proposition 5 , however with the distinction of tracking down the dependence on $p, \phi^{*}$ and $J$ wherever it appears. 
For every $\tau \in(0,1)$ set

$$
m_{\tau}(t, \lambda)=(t \lambda)^{\tau} e^{-t \lambda} m(\lambda), \quad t>0, \quad \lambda \in \mathbf{S}_{\phi^{*}},
$$

and denote by $s \longmapsto \mathscr{M}_{s} m_{\tau}$ the Mellin transform of $m_{\tau}$ with respect to the second variable. Explicitly, for $t>0$,

$$
\left[\mathscr{M}_{s} m_{\tau}\right](t)=\int_{0}^{\infty} m_{\tau}(t, \lambda) \lambda^{-i s} \frac{\mathrm{d} \lambda}{\lambda}, \quad s \in \mathbb{R} .
$$

Lemma 6. Let $\tau \in(0,1), s \in \mathbb{R}, \alpha \geqslant 0, \phi^{*} \in[0, \pi / 2)$ and $m \in H^{\infty}\left(\mathbf{S}_{\phi^{*}} ; \alpha\right)$. Then

$$
\left\|\mathscr{M}_{s} m_{\tau}\right\|_{L^{\infty}\left(\mathbb{R}_{+}\right)} \leqslant C(\alpha) \tau^{-1}\left(\cos \phi^{*}\right)^{-\tau-\alpha}(1+|s|)^{-\alpha} e^{-\phi^{*}|s|}\|m\|_{\phi^{*} ; \alpha}
$$

Proof. Suppose for a moment that $\alpha \in \mathbb{N}$. Then the lemma follows by modifying [35, Theorem 4] as described in the proof of [23, Theorem 2.2]. In order to prove the lemma for noninteger values of $\alpha$, just observe that by a simple argument of interpolation between Sobolev spaces the conclusion of [35, Theorem 4] still holds if $\alpha \in \mathbb{R}_{+} \backslash \mathbb{N}$.

Following the proof of [16, Theorem 2.1] we arrive at the representation formula

$$
m(\mathcal{A}) f=\frac{2^{\tau}}{\pi \Gamma(\tau+1)} \int_{-\infty}^{+\infty}\left[\widetilde{\mathscr{M}_{s} m_{\tau}}\right](\mathcal{A}) \mathcal{A}^{i s} f \mathrm{~d} s, \quad f \in \mathrm{R}\left(\mathcal{A}_{p}\right),
$$

where $\Gamma$ denotes the Euler's function.

By Cowling's multiplier theorem [13, Theorem 3], Laplace transform type multipliers belong to $\mathcal{M}_{p}(\mathcal{A})$. Denote by $C_{2}=C_{2}(p)$ the best constant in

$$
\|\widetilde{M}(\mathcal{A}) f\|_{p} \leqslant C\|M\|_{\infty}\|f\|_{p} \quad \forall f \in \mathrm{R}\left(\mathcal{A}_{p}\right), \forall M \in L^{\infty}\left(\mathbb{R}_{+}\right) .
$$

We can now easily obtain the following "quantitative" version of Proposition 5 .

Proposition 7. Under the assumptions of Proposition 5,

$$
\|m(\mathcal{A}) f\|_{p} \leqslant C_{1}\|f\|_{p}, \quad f \in \overline{\mathrm{R}\left(\mathcal{A}_{p}\right)},
$$

where

$$
C_{1}=C(J) C_{2}(p) \inf _{\tau \in(0,1)} \int_{-\infty}^{+\infty}\left\|\mathscr{M}_{s} m_{\tau}\right\|_{L^{\infty}\left(\mathbb{R}_{+}\right)} \cdot\left\|\mathcal{A}^{i s}\right\|_{\mathcal{B}\left(L^{p}\left(\overline{\mathrm{R}\left(\mathcal{A}_{p}\right)}, \nu\right)\right)} \mathrm{d} s
$$

is finite.

Consequently, in order to prove Theorem 1, we will first estimate the constant $C_{2}$ (see Proposition 10), and then we will estimate the growth in $s \in \mathbb{R}$ of the $L^{p}$ norms of imaginary powers $\mathcal{A}^{\text {is }}$ (see Proposition [11). To this end, we will use the following subordination formula.

For every $\vartheta \in(-\pi / 2, \pi / 2)$, consider the positively oriented path

$$
\gamma_{\vartheta}=\left\{r e^{i \vartheta}: r>0\right\}
$$

Lemma 8. Suppose that either $\psi \in(0, \pi / 2)$ and $M \in H^{\infty}\left(\mathbf{S}_{\psi}\right)$, or $\psi=0$ and $M \in$ $L^{\infty}\left(\mathbb{R}_{+}\right)$. Then, for every $\vartheta \in(-\psi, \psi) \cup\{0\}, f \in \mathrm{D}\left(\mathcal{A}_{2}\right) \cap \mathrm{R}\left(\mathcal{A}_{2}\right)$ and $g \in L^{2}(\Omega, \nu)$,

$$
\langle\widetilde{M}(\mathcal{A}) f, g\rangle=2 \int_{\gamma_{\vartheta}}\left\langle\mathcal{A} T_{z} f, T_{\bar{z}} g\right\rangle M(2 z) \mathrm{d} z .
$$

Consequently,

$$
|\langle\widetilde{M}(\mathcal{A}) f, g\rangle| \leqslant 2\|M\|_{L^{\infty}\left(\gamma_{\vartheta}\right)} \int_{\gamma_{\vartheta}}\left|\left\langle\mathcal{A} T_{z} f, T_{\bar{z}} g\right\rangle\right| \mathrm{d}|z|
$$


Proof. The lemma quickly follows from the spectral theorem, the Cauchy theorem and the fact that on $L^{2}$ we have $T_{z}^{*}=T_{\bar{z}}$.

\section{Bilinear EMbedding AND PRoof of the MUltiplier theOREM}

We now state a complex-time bilinear embedding theorem which is the principal tool for proving Theorem 1 .

If $p \in(2, \infty)$ and $\varepsilon \in(0,1 / 2)$ define

$$
p_{\varepsilon}=\frac{p-2 \varepsilon}{1-\varepsilon} \quad \text { and } \quad q_{\varepsilon}=p_{\varepsilon}^{\prime}=\frac{p_{\varepsilon}}{p_{\varepsilon}-1} .
$$

Theorem 9 (Bilinear embedding). For every $p \in(2, \infty), \varepsilon \in(0,1 / 2), \phi \in\left[-\phi_{p_{\varepsilon}}, \phi_{p_{\varepsilon}}\right]$, and all $f \in L^{p}(\Omega, \nu), g \in L^{q}(\Omega, \nu)$,

$$
\int_{\gamma_{\phi}}\left|\left\langle\mathcal{A} T_{z} f, T_{\bar{z}} g\right\rangle\right| \mathrm{d}|z| \leqslant \frac{30(p-1)}{\varepsilon \cos \phi}\|f\|_{p}\|g\|_{q} .
$$

The proof of the bilinear embedding is deferred to Section 7 Here we prove some consequences of Theorem 9, most notably Theorem 1. We first consider multipliers of Laplace transform type and, in particular, imaginary powers.

Proposition 10. Suppose that $M \in L^{\infty}\left(\mathbb{R}_{+}\right)$. Then, for every $p \in(1, \infty)$,

$$
\|\widetilde{M}(\mathcal{A}) f\|_{p} \leqslant 120\left(p^{*}-1\right)\|M\|_{\infty}\|f\|_{p}, \quad \forall f \in \overline{\mathrm{R}\left(\mathcal{A}_{p}\right)} .
$$

Proof. By duality it suffices to assume that $p>2$. The lemma follows by combining Lemma 2 (ii) with Lemma 8 and Theorem 9 applied with $\vartheta=\phi=0$ and $\varepsilon=1 / 2$.

Proposition 11. For every $s \in \mathbb{R}, p>1$ and $f \in \overline{\mathrm{R}\left(\mathcal{A}_{p}\right)}$,

$$
\left\|\mathcal{A}^{i s} f\right\|_{p} \lesssim\left(p^{*}-1\right)(1+|s|)^{1 / 2} e^{\phi_{p}^{*}|s|}\|f\|_{p} .
$$

Moreover, for every $p^{*} \geqslant 3$ and $f \in \overline{\mathrm{R}\left(\mathcal{A}_{p}\right)}$,

$$
\left\|\mathcal{A}^{i s} f\right\|_{p} \lesssim e^{\phi_{p}^{*}|s|}\|f\|_{p}\left\{\begin{array}{lll}
\left(p^{*}-1\right)(1+|s|)^{-1 / 2} & \text { if } & |s| \leqslant 2 p^{*} \sqrt{p^{*}-1} /\left(p^{*}-2\right) ; \\
\sqrt{p^{*}-1}(1+|s|)^{1 / 2} & \text { if } & |s|>2 p^{*} \sqrt{p^{*}-1} /\left(p^{*}-2\right) .
\end{array}\right.
$$

Proof. By duality it suffices to consider $p \in(2, \infty)$. Note that $\mathcal{A}^{i s}=\widetilde{M}_{s}(\mathcal{A}), s \in \mathbb{R}$, where $M_{s}(z)=\Gamma(1-i s)^{-1} z^{-i s}$ for $z \in \mathbb{C}, \Re z>0$. Evidently $M_{s} \in H^{\infty}\left(\mathbf{S}_{\vartheta}\right)$ for all $\vartheta \in(0, \pi / 2)$ and, by the asymptotic estimate $|\Gamma(1-i s)| \sim|s|^{1 / 2} e^{-\pi|s| / 2}$ as $|s| \rightarrow \infty$,

$$
\left\|M_{s}\right\|_{L^{\infty}\left(\gamma_{\vartheta}\right)} \lesssim(1+|s|)^{-1 / 2} e^{\frac{\pi|s|}{2}+s \vartheta}
$$

for all $\vartheta \in(-\pi / 2, \pi / 2)$ and $s \in \mathbb{R}$.

Fix $s \in \mathbb{R}, \varepsilon \in(0,1 / 2), r>2$ and $\phi \in\left[0, \phi_{r_{\varepsilon}}\right]$. By combining Lemma 8 (applied with $\vartheta=-\phi \operatorname{sign} s$ ) with Lemma 2 (ii) and Theorem 9, we obtain

$$
\left\|\mathcal{A}^{i s} f\right\|_{r} \lesssim \frac{r-1}{\varepsilon \cos \phi}(1+|s|)^{-1 / 2} e^{\phi^{*}|s|}\|f\|_{r} \quad f \in \overline{\mathrm{R}\left(\mathcal{A}_{r}\right)} .
$$

At this point we need to consider two cases, when $|s|$ is large and small, respectively. The decision on where to split is made according to the elementary inequality

$$
\phi_{p_{\varepsilon}}^{*} \leqslant \phi_{p}^{*}+\frac{2(p-2)}{p \sqrt{p-1}} \varepsilon \quad \forall p>2, \forall \varepsilon \in(0,1 / 2) .
$$


Suppose first that $|s|>2 p \sqrt{p-1} /(p-2)$. Then we apply (9) with $r=p$,

$$
\varepsilon=\frac{p \sqrt{p-1}}{p-2} \cdot \frac{1}{1+|s|}
$$

and $\phi=\phi_{p_{\varepsilon}}$. From (10) and the estimate $\cos \phi_{p_{\varepsilon}}>\cos \phi_{p}=1-2 / p$, we obtain

$$
\left\|\mathcal{A}^{i s} f\right\|_{p} \lesssim \sqrt{p-1}(1+|s|)^{1 / 2} e^{\phi_{p}^{*}|s|}\|f\|_{p} \quad f \in \overline{\mathrm{R}\left(\mathcal{A}_{p}\right)} .
$$

Suppose now that $|s| \leqslant 2 p \sqrt{p-1} /(p-2)$. We separate further two cases:

- If $2<p \leqslant 3$, then by interpolating the trivial $L^{2}$-estimate with (9) applied with $r=2 p, \phi=0$ and $\varepsilon=1 / 3$, we obtain

$$
\left\|\mathcal{A}^{i s} f\right\|_{p} \lesssim(p-1)\|f\|_{p} \lesssim(p-1)(1+|s|)^{1 / 2} e^{\phi_{p}^{*}|s|}\|f\|_{p} \quad f \in \overline{\mathrm{R}\left(\mathcal{A}_{p}\right)} .
$$

- If $p \geqslant 3$ then $\cos \phi_{p_{\varepsilon}} \geqslant \cos \phi_{3}=1 / 3$. Therefore, by (91) applied with $r=p$, $\varepsilon=1 / 3$ and $\phi=\phi_{p_{\varepsilon}}$, and from (10), we obtain

$$
\left\|\mathcal{A}^{i s} f\right\|_{p} \lesssim(p-1)(1+|s|)^{-1 / 2} e^{\phi_{p}^{*}|s|}\|f\|_{p} \quad f \in \overline{\mathrm{R}\left(\mathcal{A}_{p}\right)} .
$$

The proposition now follows.

Proof of Theorem 1, By combining the first inequality in Proposition 11] with Proposition [5, applied with $\sigma=1 / 2$ and $\phi^{*}=\phi_{p}^{*}$, we obtain that $m(\mathcal{A})$ is bounded on $\overline{\mathcal{R}\left(\mathcal{A}_{p}\right)}$.

We now estimate the norm of $m(\mathcal{A})$ on $\overline{\mathcal{R}\left(\mathcal{A}_{p}\right)}$ by means of Propositions 7 , By duality we may restrict ourselves to the case $p>2$. Since $\cos \phi_{p}^{*}=2 \sqrt{p-1} / p$, Lemma 6 applied first with $\phi^{*}=\phi_{p}^{*}$ and $\alpha=0$, and then with $\phi^{*}=\phi_{p}^{*}$ and $\alpha=J$ implies

$$
\left\|\mathscr{M}_{s} m_{\tau}\right\|_{L^{\infty}\left(\mathbb{R}_{+}\right)} \lesssim J \tau^{-1} p^{\tau / 2} e^{-\phi_{p}^{*}|s|} \min \left\{1,\left(\frac{\sqrt{p}}{1+|s|}\right)^{J}\right\}\|m\|_{\phi_{p}^{*} ; J} .
$$

By combining the inequality above with Proposition [10, the second estimate in Proposition 11 and (77), we eventually obtain, for any $\tau \in(0,1)$,

$$
\|m(\mathcal{A}) f\|_{p} \lesssim{ }_{J} \tau^{-1} p^{\tau / 2} p^{9 / 4}\|m\|_{\phi_{p}^{*} ; J} \quad f \in \mathrm{R}\left(\mathcal{A}_{p}\right) .
$$

To conclude the proof of the theorem, just minimize the right-hand side of the inequality above with respect to $\tau \in(0,1)$ and observe that, by Lemma 2 ,

$$
m(\mathcal{A}) f=m(0) \mathcal{P}_{0} f+m(\mathcal{A})\left(I-\mathcal{P}_{0}\right) f, \quad f \in L^{2}(\Omega, \nu) \cap L^{p}(\Omega, \nu),
$$

where $\mathcal{P}_{0}$ is a contraction on $L^{p}(\Omega, \nu)$ and $\left(I-\mathcal{P}_{0}\right) f \in \overline{\mathrm{R}\left(\mathcal{A}_{p}\right)}$.

As already remarked in the introduction, by combining our results with Cowling's subordination theory [13, Section 3], we improve his maximal theorems [13, Theorems $7,8]$ as follows. For $N \in \mathbb{N}$ set $P_{N}(\lambda)=\sum_{n=0}^{N}(-\lambda)^{n} / n$ !. For every $\delta>0, N \in \mathbb{N}$ and $\phi \in[0, \pi / 2)$ define the maximal operators

$$
\mathcal{M}^{\phi} f=\sup \left\{\left|T_{z} f\right|: z \in \overline{\mathbf{S}}_{\phi}\right\} \quad \text { and } \quad \mathcal{M}_{N, \delta}^{\phi} f=\sup \left\{|z|^{-\delta}\left|T_{z} f-P_{N}(z \mathcal{A}) f\right|: z \in \overline{\mathbf{S}}_{\phi}\right\} \text {. }
$$

Theorem 12. Suppose that $1<p<\infty$.

(i) If $0 \leqslant \phi<\phi_{p}$, then there exists $C=C(\phi, p)>0$ such that

$$
\left\|\mathcal{M}^{\phi} f\right\|_{p} \leqslant C\|f\|_{p}
$$

for all $f \in L^{2}(\Omega, \nu) \cap L^{p}(\Omega, \nu)$. 
(ii) If $\phi=\phi_{p}$, and $1 \leqslant N<\delta<N+1$, then there exists $C=C(\delta, p)>0$ such that

$$
\left\|\mathcal{M}_{N, \delta}^{\phi} f\right\|_{p} \leqslant C\left\|\mathcal{A}^{\delta} f\right\|_{p},
$$

for all $f$ in $L^{2}(\Omega, \nu)$ such that $f$ and $\mathcal{A}^{\delta} f$ are in $L^{p}(\Omega, \nu)$.

Proof. The proof is exactly as in [13. Theorems 7,8] except that one should instead of [13, Corollary 1] use Proposition [11

The theorem above, together with a standard argument of Stein [47, pp. 72-82], implies the following result, which is an improvement over [13, Corollaries 2,3].

Corollary 13. Suppose that $1<p<\infty$. If either $0 \leqslant \phi<\phi_{p}$ and $f$ is in $L^{p}(\Omega, \nu)$, or $\phi=\phi_{p}, 1 \leqslant N<\delta<N+1$ and $f, \mathcal{A} f, \ldots, \mathcal{A}^{N} f$ and $\mathcal{A}^{\delta} f$ are in $L^{p}(\Omega, \nu)$, then $T_{z} f$ converges to $f$ pointwise almost everywhere as $z$ tends to 0 in $\overline{\mathbf{S}}_{\phi}$.

\section{THE HEAT-FLOW METHOD}

In this section we illustrate the technique we will utilize for proving the bilinear embedding in Theorem 9. The exposition will be rather descriptive, aimed at giving the idea of the proof without dwelling on technical details which will be addressed later.

Fix $p \in(2, \infty)$ and $Q: \mathbb{R}^{4} \rightarrow \mathbb{R}_{+}$which is of class $C^{1}$. We shall say that the flow associated with $Q$ (and with $\mathcal{A}$ ) is regular on $L^{p}(\Omega, \nu) \times L^{q}(\Omega, \nu)$ if for every $f \in L^{p}(\Omega, \nu), g \in L^{q}(\Omega, \nu)$ and $\phi \in\left(-\phi_{p}, \phi_{p}\right)$, the function $\mathcal{E}:[0, \infty) \mapsto \mathbb{R}_{+}$, defined by

$$
\mathcal{E}(t)=\int_{\Omega} Q\left(T_{t e^{i \phi}} f, T_{t e^{-i \phi}} g\right) \mathrm{d} \nu,
$$

is continuous on $[0, \infty)$, differentiable on $(0, \infty)$ with a continuous derivative and

$$
\mathcal{E}^{\prime}(t)=\int_{\Omega} \frac{\partial}{\partial t} Q\left(T_{t e^{i \phi}} f, T_{t e^{-i \phi}} g\right) \mathrm{d} \nu
$$

We are interested in finding a nonnegative function $Q \in C^{1}\left(\mathbb{R}^{4}\right)$, possibly depending on $p$ and $\phi$, whose corresponding flow admits, for every generator $\mathcal{A}$, the following properties:

- regularity on $L^{p} \times L^{q}$;

- initial value bound, i.e., the existence of $A_{0}=A_{0}(\phi, p)>0$ such that

$$
\mathcal{E}(0) \leqslant A_{0}\left(\|f\|_{p}^{p}+\|g\|_{q}^{q}\right), \quad \forall f \in L^{p}, g \in L^{q} ;
$$

- "quantitative monotonicity", i.e., the existence of $B_{0}=B_{0}(\phi, p)>0$ such that

$$
-\mathcal{E}^{\prime}(t) \geqslant B_{0}\left|\left\langle\mathcal{A} T_{t e^{i \phi}} f, T_{t e^{-i \phi}} g\right\rangle\right|, \quad \forall f \in L^{p}, g \in L^{q}, t>0 .
$$

For if these conditions are fulfilled, then

$$
B_{0} \int_{0}^{\infty}\left|\left\langle\mathcal{A} T_{t e^{i \phi}} f, T_{t e^{-i \phi}} g\right\rangle\right| \mathrm{d} t \leqslant-\int_{0}^{\infty} \mathcal{E}^{\prime}(t) \mathrm{d} t \leqslant \mathcal{E}(0) \leqslant A_{0}\left(\|f\|_{p}^{p}+\|g\|_{q}^{q}\right) .
$$

By replacing $f$ with $\lambda f$ and $g$ with $g / \lambda$ and minimizing with respect to $\lambda>0$ the right-hand side of the inequality above, we obtain the bilinear embedding,

$$
\int_{\gamma_{\phi}}\left|\left\langle\mathcal{A} T_{z} f, T_{\bar{z}} g\right\rangle\right| \mathrm{d}|z| \leqslant C(\phi, p)\|f\|_{p}\|g\|_{q},
$$

for all $f \in L^{p}(\Omega, \nu)$ and $g \in L^{q}(\Omega, \nu)$, where $C(\phi, p)=q(p-1)^{1 / p} A_{0} / B_{0}$. 
Let us elaborate a bit on the conditions above. Write $\zeta=\left(\zeta_{1}, \zeta_{2}\right), \eta=\left(\eta_{1}, \eta_{2}\right)$ and define

$$
\partial_{\zeta}=\frac{1}{2}\left(\partial_{\zeta_{1}}-i \partial_{\zeta_{2}}\right) \quad \text { and } \quad \partial_{\eta}=\frac{1}{2}\left(\partial_{\eta_{1}}-i \partial_{\eta_{2}}\right) .
$$

Suppose that, for $\zeta, \eta \in \mathbb{R}^{2}$, the function $Q$ satisfies the following estimates:

$$
0 \leqslant Q(\zeta, \eta) \leqslant A_{0}\left(|\zeta|^{p}+|\eta|^{q}\right)
$$

and

$$
\left|\left(\partial_{\zeta} Q\right)(\zeta, \eta)\right| \lesssim|\zeta|^{p-1}+|\eta| \quad \text { and } \quad\left|\left(\partial_{\eta} Q\right)(\zeta, \eta)\right| \lesssim|\eta|^{q-1}+|\zeta| .
$$

Then (14) and (15) together with Proposition 3 give the regularity on $L^{p} \times L^{q}$ of the flow associated with $Q$. Moreover (14) clearly implies (12).

Now let us turn towards (13). We may expand (11) as

$$
\begin{aligned}
-\mathcal{E}^{\prime}(t)=2 \Re \int_{\Omega}\left[e^{i \phi}\left(\partial_{\zeta} Q\right)\left(T_{t e^{i \phi}} f, T_{t e^{-i \phi}} g\right) \mathcal{A} T_{t e^{i \phi}} f\right. \\
\left.+e^{-i \phi}\left(\partial_{\eta} Q\right)\left(T_{t e^{i \phi}} f, T_{t e^{-i \phi}} g\right) \mathcal{A} T_{t e^{-i \phi}} g\right] \mathrm{d} \nu .
\end{aligned}
$$

Thus, condition (13) can be restated as

$$
B_{0}\left|\int_{\Omega} \mathcal{A} f \cdot \bar{g} \mathrm{~d} \nu\right| \leqslant 2 \Re \int_{\Omega}\left(e^{i \phi}\left(\partial_{\zeta} Q\right)(f, g) \mathcal{A} f+e^{-i \phi}\left(\partial_{\eta} Q\right)(f, g) \mathcal{A} g\right) \mathrm{d} \nu,
$$

for all $f \in \mathrm{D}\left(\mathcal{A}_{p}\right), g \in \mathrm{D}\left(\mathcal{A}_{q}\right)$.

Convexity of $Q$ : The Riemannian case. We are interested in pointwise conditions on the second order partial derivatives of $Q$ which imply (16). We first consider the special case when $\nu=\mu$ is the Riemannian measure on a complete Riemannian manifold $\Omega=M$ and $\mathcal{A}=\Delta$ is the nonnegative Laplace-Beltrami operator on $M$.

If $\phi \in(-\pi / 2, \pi / 2)$, set

$$
\mathcal{O}_{\phi}=\left[\begin{array}{rr}
\cos \phi & -\sin \phi \\
\sin \phi & \cos \phi
\end{array}\right] \in \mathbb{R}^{2,2} \quad \text { and } \quad \mathcal{U}_{\phi}=\left[\begin{array}{ll}
\mathcal{O}_{\phi} & 0 \\
0 & \mathcal{O}_{-\phi}
\end{array}\right] \in \mathbb{R}^{4,4} .
$$

Suppose for a moment that $Q \in C^{2}\left(\mathbb{R}^{4}\right)$. Let $\mathcal{H}(Q)$ denote the Hessian matrix of $Q$. Define

$$
\mathcal{R}_{\phi}(Q)=\frac{1}{2}\left(\mathcal{U}_{\phi}^{T} \cdot \mathcal{H}(Q)+\mathcal{H}(Q) \cdot \mathcal{U}_{\phi}\right) .
$$

The proof of [48, Theorem 3.5] shows that $C_{c}^{\infty}(M)$ is a core for $\mathrm{D}\left(\Delta_{r}\right)$ when $1<r<\infty$, so that it suffices to consider (16) for $f, g \in C_{c}^{\infty}(M)$. Then, an integration by parts on the right-hand side of (16) gives

$$
2 \Re \int_{M}\left(e^{i \phi}\left(\partial_{\zeta} Q\right)(f, g) \Delta f+e^{-i \phi}\left(\partial_{\eta} Q\right)(f, g) \Delta g\right) \mathrm{d} \mu=\int_{M} \mathcal{R}_{\phi}(Q)[(f, g) ;(\mathrm{d} f, \mathrm{~d} g)] \mathrm{d} \mu,
$$

where $\mathrm{d}$ denotes the differential (there is a small yet obvious abuse of notation as to the way the identity (5) was used above). We remark that a similar identity is valid also in the case when $\Delta$ is the Ornstein-Uhlenbeck operator on a Wiener space.

It is from here not difficult to see that we could prove (16) if we had the following property of $Q$ : there exist $B_{0}=B_{0}(p, \phi)>0$ and $\tau: \mathbb{R}^{4} \rightarrow(0, \infty)$ such that for all $\xi \in \mathbb{R}^{4}$ and $\omega=\left(\omega_{1}, \omega_{2}\right) \in \mathbb{R}^{2} \times \mathbb{R}^{2}$,

$$
\mathcal{R}_{\phi}(Q)[\xi ; \omega] \geqslant B_{0}\left(\tau(\xi)\left|\omega_{1}\right|^{2}+\tau(\xi)^{-1}\left|\omega_{2}\right|^{2}\right) .
$$


Owing to a theorem by Treil, Volberg and the second author [17, Theorem 2.1], the inequality above is equivalent to the "weaker" property

$$
\mathcal{R}_{\phi}(Q)[\xi ; \omega] \gtrsim p, \phi\left|\omega_{1}\right|\left|\omega_{2}\right| .
$$

We can relax the condition $Q \in C^{2}\left(\mathbb{R}^{4}\right)$ by requiring that $Q$ be of class $C^{1}$ and almost everywhere twice differentiable with second-order weak derivatives. Then we can consider the flow corresponding to a regularization of $Q$ by standard mollifiers (see Corollaries 17 and 18).

When $\mathcal{A}$ is merely a generator of a symmetric contraction semigroup but not a differential-type operator, it is not so obvious that (19) implies (16). However, this is actually the case and will be proved in Section 7

When $\phi=0$, similar heat-flow techniques corresponding to Bellman functions have so far been employed in the Euclidean case [43, 41, 18, 19, 20] and recently also in the Riemannian case [10]. For a different perspective on heat-flow techniques, various examples and references we refer the reader to the papers by Bennett et al. [4, 5.

Summary. The proof of Theorem 9 reduces to finding for every $p>2$ and $\phi \in$ $\left(-\phi_{p}, \phi_{p}\right)$ a function $Q \in C^{1}\left(\mathbb{R}^{4}\right)$ such that

(i) $0 \leqslant Q(\zeta, \eta) \lesssim{ }_{p, \phi}|\zeta|^{p}+|\eta|^{q}, \quad \forall \zeta, \eta \in \mathbb{R}^{2}$;

(ii) $\left|\left(\partial_{\zeta} Q\right)(\zeta, \eta)\right| \lesssim|\zeta|^{p-1}+|\eta| \quad$ and $\quad\left|\left(\partial_{\eta} Q\right)(\zeta, \eta)\right| \lesssim|\eta|^{q-1}+|\zeta|, \quad \forall \zeta, \eta \in \mathbb{R}^{2}$;

(iii) the function $Q$ is almost everywhere twice differentiable with second-order weak derivatives and

$$
\mathcal{R}_{\phi}(Q)[\xi ; \omega] \gtrsim p, \phi\left|\omega_{1}\right|\left|\omega_{2}\right|,
$$

for almost every $\xi \in \mathbb{R}^{4}$ and all $\omega=\left(\omega_{1}, \omega_{2}\right) \in \mathbb{R}^{2} \times \mathbb{R}^{2}$.

It is not an easy matter to find functions $Q$ satisfying (i), (ii) and (iii) with "optimal" constants, even when $\phi=0$. It turned out [38, 19] that, if $\phi=0$, we can take for $Q$ the so-called Nazarov-Treil Bellman function which will be defined in the next section. We show in this paper that the very same Nazarov-Treil Bellman function satisfies the condition (iii) in the whole range of $\phi$ we are interested in, i.e., $\left(-\phi_{p}, \phi_{p}\right)$; see Theorem 15 for the precise statement.

\section{Nazarov-Treil Bellman function}

In this section we fix $p \in(2, \infty)$ and $\varepsilon \in(0,1 / 2)$. Set

$$
\delta=\delta(p, \varepsilon)=\frac{2 q(q-1) \varepsilon}{85}
$$

and define the Nazarov-Treil Bellman function $Q: \mathbb{R}^{2} \times \mathbb{R}^{2} \longrightarrow \mathbb{R}_{+}$by

$$
Q(\zeta, \eta)=|\zeta|^{p}+|\eta|^{q}+\delta \begin{cases}|\zeta|^{2}|\eta|^{2-q} & ;|\zeta|^{p} \leqslant|\eta|^{q} \\ \frac{2}{p}|\zeta|^{p}+\left(\frac{2}{q}-1\right)|\eta|^{q} & ;|\zeta|^{p} \geqslant|\eta|^{q} .\end{cases}
$$

The reasons for the particular choice of $\delta$ in (20) will only become apparent later; see Remark 25,

The origins of $Q$ lie in the paper of F. Nazarov and S. Treil [38]. A modification of their function was later applied by A. Volberg and the second author in [18, 19]. Here we use a simplified variant which comprises only two variables. It was introduced in [20] and used by the present authors in [10]. The function $Q$ above is essentially the 
same as in [20, 10], except for the fact that here $\delta$ also depends on a new parameter $\varepsilon$.

The construction of the original Nazarov-Treil function in 38 was one of the earliest examples of the so-called Bellman function technique, which was introduced in harmonic analysis shortly beforehand by Nazarov, Treil and Volberg [39]. Earlier, the Bellman functions have implicitly appeared in the work of Burkholder [6], see also [7, 8]. If interested in the genesis of Bellman functions and the overview of the method, the reader is also referred to Volberg et al. [40, 53, 38, and Wittwer [54]. The method has seen a whole series of applications, mostly in Euclidean harmonic analysis.

In the course of the last few years, the Nazarov-Treil function considered here was found to possess nontrivial properties that reach much beyond the need for which it was originally constructed in [38. These properties are used for proving several variants of the bilinear embedding. For example, such was the case in [19, 20] with general Schrödinger operators. As already mentioned, in the present paper we shall prove another convexity property of $Q$ (see Theorem 15).

The following result is a direct consequence of the definition of $Q$.

Proposition 14. The function $Q$ belongs to $C^{1}\left(\mathbb{R}^{4}\right)$ and is of order $C^{2}$ everywhere except on the set $\Upsilon_{0}=\left\{(\zeta, \eta) \in \mathbb{R}^{2} \times \mathbb{R}^{2}:(\eta=0) \vee\left(|\zeta|^{p}=|\eta|^{q}\right)\right\}$.

For every $(\zeta, \eta) \in \mathbb{R}^{2} \times \mathbb{R}^{2}$,

$$
0 \leqslant Q(\zeta, \eta) \leqslant(1+\delta)\left(|\zeta|^{p}+|\eta|^{q}\right),
$$

and

$$
\begin{aligned}
& 2\left|\left(\partial_{\zeta} Q\right)(\zeta, \eta)\right| \leqslant(p+2 \delta) \max \left\{|\zeta|^{p-1},|\eta|\right\}, \\
& 2\left|\left(\partial_{\eta} Q\right)(\zeta, \eta)\right| \leqslant(q+(2-q) \delta)|\eta|^{q-1} .
\end{aligned}
$$

Next result is one of the key estimates of the paper and will be proved in Section 6 . Recall that $p_{\varepsilon}, q_{\varepsilon}$ and $\mathcal{R}_{\phi}$ were defined in (8) and (18), respectively. In particular, by (5),

$$
\mathcal{R}_{\phi}(Q)[\xi ; \omega]=\left\langle\mathcal{H}(Q)(\xi) \omega, \mathcal{U}_{\phi} \omega\right\rangle_{\mathbb{R}^{4}} .
$$

Theorem 15. For every $\phi \in\left[-\phi_{p_{\varepsilon}}, \phi_{p_{\varepsilon}}\right]$,

$$
\mathcal{R}_{\phi}(Q)[\xi ; \omega] \geqslant 2 \delta \cos \phi\left|\omega_{1}\right|\left|\omega_{2}\right|,
$$

for all $\xi \in\left(\mathbb{R}^{2} \times \mathbb{R}^{2}\right) \backslash \Upsilon_{0}$ and $\omega=\left(\omega_{1}, \omega_{2}\right) \in \mathbb{R}^{2} \times \mathbb{R}^{2}$.

Remark 16. The special case $\phi=0$ follows from earlier papers, see [38, Section 8] and [19, Theorem 3]. Actually, the estimate for $\phi=0$ was essentially the very purpose of constructing the prototype of $Q$ in 38 .

Regularization of $Q$. Denote by $*$ convolution in $\mathbb{R}^{4}$, and let $\left(\psi_{r}\right)_{r>0}$ be a nonnegative, smooth and compactly supported approximation to the identity in $\mathbb{R}^{4}$. Since $Q \in C^{1}\left(\mathbb{R}^{4}\right)$ and its second-order partial derivatives exist on $\mathbb{R}^{4} \backslash \Upsilon_{0}$ and are locally integrable in $\mathbb{R}^{4}$,

$$
\mathcal{R}_{\phi}\left(Q * \psi_{r}\right)[\xi ; \omega]=\int_{\mathbb{R}^{4}} \mathcal{R}_{\phi}(Q)\left[\xi-\xi^{\prime} ; \omega\right] \psi_{r}\left(\xi^{\prime}\right) \mathrm{d} \xi^{\prime}
$$

for every $r>0, \xi \in \mathbb{R}^{4}$ and every $\omega=\left(\omega_{1}, \omega_{2}\right) \in \mathbb{R}^{2} \times \mathbb{R}^{2}$. 
Corollary 17. For every $r>0$ and $\phi \in\left[-\phi_{p_{\varepsilon}}, \phi_{p_{\varepsilon}}\right]$,

$$
\mathcal{R}_{\phi}\left(Q * \psi_{r}\right)[\xi ; \omega] \geqslant 2 \delta \cos \phi\left|\omega_{1}\right|\left|\omega_{2}\right|,
$$

for all $\xi \in \mathbb{R}^{4}$ and every $\omega=\left(\omega_{1}, \omega_{2}\right) \in \mathbb{R}^{2} \times \mathbb{R}^{2}$.

Proof. The corollary immediately follows from (22) and Theorem 15 ,

For use later in Section 7 we state the main corollary of Theorem 15, If $\alpha, \beta \in \mathbb{C}$ and $\phi \in(-\pi / 2, \pi / 2)$, define $F_{\alpha, \beta}: \mathbb{C}^{2} \rightarrow \mathbb{R}$ by the rule

$$
F_{\alpha, \beta}(\xi)=2 \Re\left[e^{i \phi} \alpha \cdot\left(\partial_{\zeta} Q\right)(\xi)+e^{-i \phi} \beta \cdot\left(\partial_{\eta} Q\right)(\xi)\right] .
$$

Corollary 18. Let $\alpha, \beta \in \mathbb{C}$. Then, for every $\phi \in\left[-\phi_{p_{\varepsilon}}, \phi_{p_{\varepsilon}}\right]$, and all $\zeta, \eta \in \mathbb{C}$,

$$
F_{\alpha, \beta}(\alpha+\zeta, \beta+\eta)-F_{\alpha, \beta}(\zeta, \eta) \geqslant 2 \delta \cos \phi|\alpha||\beta| .
$$

Proof. For every $r>0$, let $F_{r, \alpha, \beta}$ be the function defined by replacing $Q$ with $Q * \psi_{r}$ in (24). Since $Q$ is $C^{1}$, one has $\lim _{r \rightarrow 0^{+}} F_{r, \alpha, \beta}(\xi)=F_{\alpha, \beta}(\xi)$ for all $\xi \in \mathbb{C}^{2}$. Therefore, it suffices to prove the lemma with $F_{\alpha, \beta}$ replaced by $F_{r, \alpha, \beta}$.

If $u=\left(u_{1}, u_{2}\right)$ is a vector in $\mathbb{C}^{2}$, let us denote by $\mathcal{V}(u)$ its real counterpart in $\mathbb{R}^{4}$, that is,

$$
\mathcal{V}(u)=\left(\Re u_{1}, \Im u_{1}, \Re u_{2}, \Im u_{2}\right) .
$$

Whit this notation, we have

$$
F_{r, \alpha, \beta}(\xi)=\left\langle\mathcal{U}_{\phi} \cdot \mathcal{V}(\alpha, \beta), \nabla\left(Q * \psi_{r}\right)(\xi)\right\rangle_{\mathbb{R}^{4}} .
$$

Therefore, for all $\xi \in \mathbb{C}^{2}$,

$$
\left\langle\mathcal{V}(\alpha, \beta), \nabla F_{r, \alpha, \beta}(\xi)\right\rangle_{\mathbb{R}^{4}}=\mathcal{R}_{\phi}\left(Q * \psi_{r}\right)[\xi ; \mathcal{V}(\alpha, \beta)] .
$$

The result now follows from the mean value theorem and Corollary 17.

\section{Proof of Theorem 15}

We shall throughout this section keep in mind that $p>2$ and $\varepsilon \in(0,1 / 2]$ are fixed numbers, that $q=p /(p-1)$ is the conjugate exponent of $p$ and that $p_{\varepsilon}, q_{\varepsilon}$ were defined in (8). Furthermore, recall that with $p, \varepsilon$ as above we introduced $\delta=\delta(p, \varepsilon)$ in (20) and that the function $Q=Q_{p, \varepsilon}$ was defined in (21) by means of these $p, \delta$. Finally, bear in mind that $\mathcal{H}(Q)$ denotes the Hessian matrix of $Q$, and that, for $\phi \in(-\pi / 2, \pi / 2)$, the matrix function $\mathcal{R}_{\phi}(Q)$ was defined in (18). We can write

$$
\mathcal{R}_{\phi}(Q)=\cos \phi \cdot \mathcal{H}(Q)+\sin \phi \cdot \mathcal{J}(Q)
$$

with the matrices $\mathcal{H}(Q)$ and $\mathcal{J}(Q)$ expressed in the block matrix notation as

$$
\mathcal{H}(Q)=\left[\begin{array}{ll}
\mathcal{H}_{1}(Q) & \mathcal{H}_{2}(Q) \\
\mathcal{H}_{2}^{T}(Q) & \mathcal{H}_{3}(Q)
\end{array}\right] \quad \text { and } \quad \mathcal{J}(Q)=\left[\begin{array}{cc}
\mathcal{J}_{1}(Q) & \mathcal{J}_{2}(Q) \\
\mathcal{J}_{2}^{T}(Q) & -\mathcal{J}_{3}(Q)
\end{array}\right],
$$

where, in turn, $\mathcal{J}_{j}(Q)$ are $2 \times 2$ real matrices given by

$$
\begin{aligned}
& \mathcal{J}_{1}(Q)=\left[\begin{array}{cc}
\partial_{\zeta_{1} \zeta_{2}}^{2} Q & \frac{1}{2}\left(\partial_{\zeta_{2} \zeta_{2}}^{2} Q-\partial_{\zeta_{1} \zeta_{1}}^{2} Q\right) \\
\frac{1}{2}\left(\partial_{\zeta_{2} \zeta_{2}}^{2} Q-\partial_{\zeta_{1} \zeta_{1}}^{2} Q\right) & -\partial_{\zeta_{1} \zeta_{2}}^{2} Q
\end{array}\right], \\
& \mathcal{J}_{3}(Q)=\left[\begin{array}{cc}
\partial_{\eta_{1} \eta_{2}}^{2} Q & \frac{1}{2}\left(\partial_{\eta_{2} \eta_{2}}^{2} Q-\partial_{\eta_{1} \eta_{1}}^{2} Q\right) \\
\frac{1}{2}\left(\partial_{\eta_{2} \eta_{2}}^{2} Q-\partial_{\eta_{1} \eta_{1}}^{2} Q\right) & -\partial_{\eta_{1} \eta_{2}}^{2} Q
\end{array}\right]
\end{aligned}
$$


and

$$
\mathcal{J}_{2}(Q)=\frac{1}{2}\left[\begin{array}{rr}
\partial_{\zeta_{2} \eta_{1}}^{2} Q-\partial_{\zeta_{1} \eta_{2}}^{2} Q & \partial_{\zeta_{1} \eta_{1}}^{2} Q+\partial_{\zeta_{2} \eta_{2}}^{2} Q \\
-\partial_{\zeta_{1} \eta_{1}}^{2} Q-\partial_{\zeta_{2} \eta_{2}}^{2} Q & \partial_{\zeta_{2} \eta_{1}}^{2} Q-\partial_{\zeta_{1} \eta_{2}}^{2} Q
\end{array}\right] .
$$

Proving Theorem 15 requires several steps. Given $\xi \in\left(\mathbb{R}^{2} \times \mathbb{R}^{2}\right) \backslash \Upsilon_{0}$ and $\omega=$ $\left(\omega_{1}, \omega_{2}\right) \in \mathbb{R}^{2} \times \mathbb{R}^{2}$, we have

$$
\begin{aligned}
(\cos \phi)^{-1} \mathcal{R}_{\phi}(Q)[\xi ; \omega] & =\left\langle\left(\mathcal{H}_{1}(Q)+\tan \phi \cdot \mathcal{J}_{1}(Q)\right)(\xi) \omega_{1}, \omega_{1}\right\rangle_{\mathbb{R}^{2}} \\
+ & 2\left\langle\left(\mathcal{H}_{2}(Q)+\tan \phi \cdot \mathcal{J}_{2}(Q)\right)(\xi) \omega_{1}, \omega_{2}\right\rangle_{\mathbb{R}^{2}} \\
& +\left\langle\left(\mathcal{H}_{3}(Q)-\tan \phi \cdot \mathcal{J}_{3}(Q)\right)(\xi) \omega_{2}, \omega_{2}\right\rangle_{\mathbb{R}^{2}}
\end{aligned}
$$

We shall split the region $\left(\mathbb{R}^{2} \times \mathbb{R}^{2}\right) \backslash \Upsilon_{0}$ into a "good" and a "bad" part as $\Omega_{g}=\left\{(\zeta, \eta) \in \mathbb{R}^{2} \times \mathbb{R}^{2}:|\zeta|^{p}>|\eta|^{q}>0\right\} \quad$ and $\quad \Omega_{b}=\left\{(\zeta, \eta) \in \mathbb{R}^{2} \times \mathbb{R}^{2}:|\zeta|^{p}<|\eta|^{q}\right\}$.

In both regions above we estimate the three terms on the right-hand side of (25) one by one. The obtained results will be summarized in Propositions 20 and 24, respectively, and Theorem 15 will then follow by combining them.

Power functions. Define, for $v \in \mathbb{R}^{2}$ and $r>1$,

$$
F_{r}(v)=|v|^{r} .
$$

The Nazarov-Treil function $Q$ essentially comprises three tensor products of power functions, namely $F_{p} \otimes \mathbf{1}, \mathbf{1} \otimes F_{q}$ and $F_{2} \otimes F_{2-q}$. Our goal is to study the quadratic form on $\mathbb{R}^{4}$ generated by the matrix $\mathcal{H}(Q) \mathcal{U}_{\phi}$, with $\mathcal{U}_{\phi}$ as in (17). We have, for example, $\mathcal{H}\left(F_{p} \otimes \mathbf{1}\right) \mathcal{U}_{\phi}=\left[\mathcal{H}\left(F_{p}\right) \mathcal{O}_{\phi}\right] \oplus \mathbf{0}$. Hence it is useful to understand first the properties of the quadratic forms on $\mathbb{R}^{2}$ induced by the matrices $\mathcal{H}\left(F_{r}\right) \mathcal{O}_{\phi}, r>1$. They have been earlier studied by Bakry 3. Define

$$
S_{r, \phi}(v)=\frac{1}{2}\left(\mathcal{H}\left(F_{r}\right)(v) \mathcal{O}_{\phi}+\mathcal{O}_{-\phi} \mathcal{H}\left(F_{r}\right)(v)\right) .
$$

A calculation shows that

$$
S_{r, \phi}(v)=r|v|^{r-2} \cos \phi \cdot \mathcal{D}_{r, \phi}(v),
$$

where

$$
\mathcal{D}_{r, \phi}(v)=\frac{r}{2}\left(I_{2}+\frac{1-2 / r}{\cos \phi} \mathcal{K}(2 \varphi-\phi)\right)
$$

with

$$
\mathcal{K}(\alpha)=\left[\begin{array}{rr}
1 & 0 \\
0 & -1
\end{array}\right] \mathcal{O}_{-\alpha}=\left[\begin{array}{rr}
\cos \alpha & \sin \alpha \\
\sin \alpha & -\cos \alpha
\end{array}\right],
$$

and $\varphi$ being the polar angle of $v$.

Lemma 19 (3]). Let $\phi \in(-\pi / 2, \pi / 2)$ and $r>1$. For every $v \in \mathbb{R}^{2} \backslash\{0\}$, the matrix $\mathcal{D}_{r, \phi}(v)$ induces a positive semidefinite quadratic form on $\mathbb{R}^{2}$ if and only if $|\phi| \leqslant \phi_{r}$.

Proof. Note that $\operatorname{tr} \mathcal{D}_{r, \phi}(v)=r>0$ and $\operatorname{det} \mathcal{D}_{r, \phi}(v)=(r / 2)^{2}\left[1-\left(\cos \phi_{r} / \cos \phi\right)^{2}\right]$, which is nonnegative if and only if $|\phi| \leqslant \phi_{r}$. 
In the continuation we use Lemma 19 for estimating the terms in (25). We also need the following identities which can be easily deduced from the very definition of $\mathcal{D}_{r, \phi}$.

$$
\begin{aligned}
& \mathcal{D}_{p, \phi}(v)=\varepsilon I_{2}+(1-\varepsilon) \mathcal{D}_{p_{\varepsilon}, \phi}(v), \\
& \mathcal{D}_{q, \phi}(v)=\varepsilon(q-1) I_{2}+[1-\varepsilon(q-1)] \mathcal{D}_{q_{\varepsilon}, \phi}(v), \\
& (2-q) \mathcal{D}_{2-q, \phi}(v)=-2(q-1) I_{2}+q \mathcal{D}_{q, \phi}(v) .
\end{aligned}
$$

Proposition 20. For all $\xi \in \Omega_{g}, \omega=\left(\omega_{1}, \omega_{2}\right) \in \mathbb{R}^{2} \times \mathbb{R}^{2}$ and $\phi \in\left[-\phi_{p_{\varepsilon}}, \phi_{p_{\varepsilon}}\right]$ we have

$$
\mathcal{R}_{\phi}(Q)[\xi ; \omega] \geqslant 2 \delta \cos \phi\left|\omega_{1}\right|\left|\omega_{2}\right| \text {. }
$$

Proof. First note that in the region $\Omega_{g}$ we have $\mathcal{H}_{2}(Q)=\mathfrak{J}_{2}(Q)=0$. As for the other blocks on the right-hand side of (25),

$$
\begin{aligned}
& \left(\mathcal{H}_{1}(Q)+\tan \phi \cdot \mathcal{J}_{1}(Q)\right)(\zeta, \eta)=(p+2 \delta)|\zeta|^{p-2} \mathcal{D}_{p, \phi}(\zeta) \\
& \left(\mathcal{H}_{3}(Q)-\tan \phi \cdot \mathcal{J}_{3}(Q)\right)(\zeta, \eta)=(q+(2-q) \delta)|\eta|^{q-2} \mathcal{D}_{q,-\phi}(\eta) .
\end{aligned}
$$

By Lemma 19, the quadratic forms $\mathcal{D}_{p_{\varepsilon}, \phi}(\zeta)$ and $\mathcal{D}_{q_{\varepsilon},-\phi}(\eta)$ are positive semidefinite, because $|\phi| \leqslant \phi_{p_{\varepsilon}}=\phi_{q_{\varepsilon}}$ by assumption. Therefore, by (26) and (27),

$$
\begin{aligned}
& \left(\mathcal{H}_{1}(Q)+\tan \phi \cdot \mathcal{J}_{1}(Q)\right)(\zeta, \eta) \geqslant \varepsilon p|\zeta|^{p-2} I_{2} \\
& \left(\mathcal{H}_{3}(Q)-\tan \phi \cdot \mathcal{J}_{3}(Q)\right)(\zeta, \eta) \geqslant \varepsilon(q-1) q|\eta|^{q-2} I_{2} .
\end{aligned}
$$

It follows from (25) that

$$
\mathcal{R}_{\phi}(Q)[\xi ; \omega] \geqslant \cos \phi\left(\varepsilon p|\zeta|^{p-2}\left|\omega_{1}\right|^{2}+\varepsilon q(q-1)|\eta|^{q-2}\left|\omega_{2}\right|^{2}\right) .
$$

Since in $\Omega_{g}$ one has that $|\eta|^{q-2}>|\zeta|^{2-p}$, we can at this point quickly finish the proof.

We now estimate the terms on the right-hand side of (25) in the bad region $\Omega_{b}$. Define

so that in $\Omega_{b}$ we have

$$
G(\zeta, \eta)=|\zeta|^{p}+|\eta|^{q} \quad \text { and } \quad H(\zeta, \eta)=|\zeta|^{2}|\eta|^{2-q}
$$

$$
Q=G+\delta H .
$$

Lemma 21. For every $\xi=(\zeta, \eta) \in \Omega_{b}, \omega_{1} \in \mathbb{R}^{2}$ and $\phi \in\left[-\phi_{p}, \phi_{p}\right]$ one has

$$
\left\langle\left(\mathcal{H}_{1}(Q)+\tan \phi \cdot \mathcal{J}_{1}(Q)\right)(\xi) \omega_{1}, \omega_{1}\right\rangle_{\mathbb{R}^{2}} \geqslant 2 \delta|\eta|^{2-q}\left|\omega_{1}\right|^{2} .
$$

Proof. We have

$$
\left(\mathcal{H}_{1}(G)+\tan \phi \cdot \mathcal{J}_{1}(G)\right)(\zeta, \eta)=p|\zeta|^{p-2} \mathcal{D}_{p, \phi}(\zeta)
$$

as well as

$$
\mathcal{H}_{1}(H)(\zeta, \eta)=2|\eta|^{2-q} I_{2} \quad \text { and } \quad \mathcal{J}_{1}(H)(\zeta, \eta)=0 .
$$

The required estimate now follows from (29) and Lemma 19.

Lemma 22. For every $\xi \in \Omega_{b}, \omega_{1}, \omega_{2} \in \mathbb{R}^{2}$ and $\phi \in\left[-\phi_{p}, \phi_{p}\right]$ one has

$$
\left\langle\left(\mathcal{H}_{2}(Q)+\tan \phi \cdot \mathcal{J}_{2}(Q)\right)(\xi) \omega_{1}, \omega_{2}\right\rangle_{\mathbb{R}^{2}} \geqslant-8 \delta\left|\omega_{1}\right|\left|\omega_{2}\right| .
$$

Proof. Observe that $\mathcal{H}_{2}(Q)+\tan \phi \cdot \mathcal{J}_{2}(Q)=\delta\left(\mathcal{H}_{2}(H)+\tan \phi \cdot \mathcal{J}_{2}(H)\right)$. Since

$$
\left(\partial_{\zeta_{i} \eta_{j}}^{2} H\right)(\zeta, \eta)=2(2-q) \zeta_{i} \eta_{j}|\eta|^{-q}
$$

the absolute values of the entries of $\mathcal{H}_{2}(H)$ and $\mathcal{J}_{2}(H)$ are uniformly bounded in $\Omega_{b}$ by $2(2-q)$. Consequently,

$$
\left\langle\left(\mathcal{H}_{2}(Q)+\tan \phi \cdot \mathcal{J}_{2}(Q)\right)(\xi) \omega_{1}, \omega_{2}\right\rangle_{\mathbb{R}^{2}} \geqslant-4 \delta(2-q)(1+|\tan \phi|)\left|\omega_{1}\right|\left|\omega_{2}\right| .
$$


We can now use the assumption on $\phi$ and estimate

$$
(2-q)(1+|\tan \phi|) \leqslant(2-q)\left(1+\tan \phi_{p}\right)=2-q+\frac{2}{\sqrt{p-1}}<2 .
$$

This finishes the proof of Lemma 22

Lemma 23. For every $\xi=(\zeta, \eta) \in \Omega_{b}, \omega_{2} \in \mathbb{R}^{2}$ and $\phi \in\left[-\phi_{p_{\varepsilon}}, \phi_{p_{\varepsilon}}\right]$,

$$
\left\langle\left(\mathcal{H}_{3}(Q)-\tan \phi \cdot \mathcal{J}_{3}(Q)\right)(\xi) \omega_{2}, \omega_{2}\right\rangle_{\mathbb{R}^{2}} \geqslant \frac{81}{2} \delta|\eta|^{q-2}\left|\omega_{2}\right|^{2} .
$$

Proof. We start by writing

$$
\begin{aligned}
\left(\mathcal{H}_{3}(G)-\tan \phi \cdot \mathcal{J}_{3}(G)\right)(\zeta, \eta) & =q|\eta|^{q-2} \mathcal{D}_{q,-\phi}(\eta), \\
\left(\mathcal{H}_{3}(H)-\tan \phi \cdot \mathcal{J}_{3}(H)\right)(\zeta, \eta) & =(2-q)|\zeta|^{2}|\eta|^{-q} \mathcal{D}_{2-q,-\phi}(\eta) .
\end{aligned}
$$

Since the determinant of the matrix $\mathcal{D}_{2-q,-\phi}(\eta)$ is negative, we consider two cases.

Case 1: Suppose that $\left\langle\mathcal{D}_{2-q,-\phi}(\eta) \omega_{2}, \omega_{2}\right\rangle_{\mathbb{R}^{2}} \geqslant 0$. Then from (29), (30) and (27) we get

$$
\begin{gathered}
\left\langle\left(\mathcal{H}_{3}(Q)-\tan \phi \cdot \mathcal{J}_{3}(Q)\right)(\zeta, \eta) \omega_{2}, \omega_{2}\right\rangle_{\mathbb{R}^{2}} \geqslant\left\langle\left(\mathcal{H}_{3}(G)-\tan \phi \cdot \mathcal{J}_{3}(G)\right)(\zeta, \eta) \omega_{2}, \omega_{2}\right\rangle_{\mathbb{R}^{2}} \\
=q(q-1) \varepsilon|\eta|^{q-2}\left|\omega_{2}\right|^{2}+q[1-\varepsilon(q-1)]|\eta|^{q-2}\left\langle\mathcal{D}_{q_{\varepsilon},-\phi}(\eta) \omega_{2}, \omega_{2}\right\rangle_{\mathbb{R}^{2}} .
\end{gathered}
$$

The required estimate now follows since the last term in the right-hand side is nonnegative by Lemma 19, because $|\phi| \leqslant \phi_{p_{\varepsilon}}=\phi_{q_{\varepsilon}}$.

Case 2: Suppose that $\left\langle\mathcal{D}_{2-q,-\phi}(\eta) \omega_{2}, \omega_{2}\right\rangle_{\mathbb{R}^{2}} \leqslant 0$. Since in $\Omega_{b}$ we have $|\zeta|^{2}<|\eta|^{2 q-2}$, identities (29), (30) imply

$$
\begin{aligned}
\left\langle\left(\mathcal{H}_{3}(Q)-\tan \phi \cdot \mathcal{J}_{3}(Q)\right)(\zeta, \eta) \omega_{2}, \omega_{2}\right\rangle_{\mathbb{R}^{2}} & \\
& \geqslant|\eta|^{q-2}\left(q\left\langle\mathcal{D}_{q,-\phi}(\eta) \omega_{2}, \omega_{2}\right\rangle_{\mathbb{R}^{2}}+\delta(2-q)\left\langle\mathcal{D}_{2-q,-\phi}(\eta) \omega_{2}, \omega_{2}\right\rangle_{\mathbb{R}^{2}}\right) .
\end{aligned}
$$

From (28) we get

$$
q \mathcal{D}_{q,-\phi}(\eta)+\delta(2-q) \mathcal{D}_{2-q,-\phi}(\eta)=q(\delta+1) \mathcal{D}_{q,-\phi}(\eta)-2 \delta(q-1) I_{2} .
$$

By Lemma 19 the quadratic form $\mathcal{D}_{q_{\varepsilon}, \phi}(\eta)$ is nonnegative, because $|\phi| \leqslant \phi_{p_{\varepsilon}}=\phi_{q_{\varepsilon}}$. Therefore, it follows from (27) and from the definition of $\delta(20)$ that

$$
\begin{aligned}
\left\langle\left(\mathcal{H}_{3}(Q)-\tan \phi \cdot \mathcal{J}_{3}(Q)\right)(\zeta, \eta) \omega_{2}, \omega_{2}\right\rangle_{\mathbb{R}^{2}} & \geqslant(q(q-1) \varepsilon(\delta+1)-2 \delta(q-1))|\eta|^{q-2}\left|\omega_{2}\right|^{2} \\
& \geqslant \frac{81}{2} \delta|\eta|^{q-2}\left|\omega_{2}\right|^{2},
\end{aligned}
$$

which concludes the proof of the lemma.

Proposition 24. For all $\xi \in \Omega_{b}, \omega=\left(\omega_{1}, \omega_{2}\right) \in \mathbb{R}^{2} \times \mathbb{R}^{2}$ and $\phi \in\left[-\phi_{p_{\varepsilon}}, \phi_{p_{\varepsilon}}\right]$ we have

$$
\mathcal{R}_{\phi}(Q)[\xi ; \omega] \geqslant 2 \delta \cos \phi\left|\omega_{1}\right|\left|\omega_{2}\right| .
$$

Proof. Combine (25) with Lemmas 21, 22 and 23.

Remark 25. Let us explain why $\delta$ was chosen in (20) the way it was.

By taking for $\delta$ a number of the form $D q(q-1) \varepsilon$ for some $\varepsilon>0$ to be determined, the proofs of Lemmas 21 and 22 would not change at all, while in Lemma 23 we would get $\langle\cdot\rangle \geqslant(1 / D-2) \delta|\eta|^{q-2}\left|\omega_{2}\right|^{2}$, and consequently in Proposition 24 we would get

$$
\mathcal{R}_{\phi}(Q)[\xi ; \omega] \geqslant(\underbrace{2 \sqrt{2 / D-4}-16}_{(*)}) \delta \cos \phi\left|\omega_{1}\right|\left|\omega_{2}\right| .
$$

If we want $(*)$ to equal 2 , we must choose $D=2 / 85$. 


\section{Proof of Theorem 9}

Fix $p \in(2, \infty), \varepsilon \in(0,1 / 2)$ and define $\delta$ and $Q$ as in (20) and (21). In order to prove the bilinear embedding we apply the heat-flow method that we described in Section 4 .

The pointwise estimates of $Q$ and its partial derivatives (see Proposition 14) in particular imply that $Q$ satisfies (14) and (15), so that the associated flow $\mathcal{E}$ is regular on $L^{p}(\Omega, \nu) \times L^{q}(\Omega, \nu)$. Moreover, $\mathcal{E}$ satisfies (12) with $A_{0}=1+\delta$, for all $\phi \in\left[-\phi_{p_{\varepsilon}}, \phi_{p_{\varepsilon}}\right]$. Therefore, what is left to prove is that $\mathcal{E}$ satisfies (13) or, equivalently, that $\mathcal{A}$ satisfies (16) for an appropriate $B_{0}>0$. In this section (see Corollary 33) we shall prove that (16) holds with $B_{0}=2 \delta \cos \phi$, for any $\phi \in\left[-\phi_{p_{\varepsilon}}, \phi_{p_{\varepsilon}}\right]$; Theorem 9 will follow immediately.

We first consider the special case when $\mathcal{A}$ is the heat generator on the two-point space, i.e.,

$$
\mathcal{A}=\mathcal{G}=\left[\begin{array}{rr}
1 & -1 \\
-1 & 1
\end{array}\right]
$$

acting on $\mathbb{C}^{2}=L^{\infty}\left(\{a, b\}, \nu_{a, b}\right)$, where $\nu_{a, b}=\left(\delta_{a}+\delta_{b}\right) / 2$. It is easy to see that $\mathcal{G}$ generates a symmetric contraction semigroup on $\left(\{a, b\}, \nu_{a, b}\right)$, e.g. [30, Example 1].

Proposition 26. For all $\phi \in\left[-\phi_{p_{\varepsilon}}, \phi_{p_{\varepsilon}}\right]$ and every $f, g:\{a, b\} \rightarrow \mathbb{C}$,

$$
2 \delta \cos \phi\left|\int_{\{a, b\}} \mathcal{G} f \cdot \bar{g} \mathrm{~d} \nu_{a, b}\right| \leqslant 2 \Re \int_{\{a, b\}}\left(e^{i \phi}\left(\partial_{\zeta} Q\right)(f, g) \mathcal{G} f+e^{-i \phi}\left(\partial_{\eta} Q\right)(f, g) \mathcal{G} g\right) \mathrm{d} \nu_{a, b} .
$$

Proof. Since for every $u, v:\{a, b\} \rightarrow \mathbb{C}$ one has

$$
\int_{\{a, b\}} \mathcal{G} u \cdot v \mathrm{~d} \nu_{a, b}=\frac{1}{2}(u(a)-u(b))(v(a)-v(b)),
$$

the proposition follows from Corollary 18 applied with $\alpha=f(a)-f(b), \beta=g(a)-g(b)$, $\zeta=f(b)$ and $\eta=g(b)$.

The general case. The idea of proving (16) in the general case is to verify first a version of (16) with $\mathcal{A}$ replaced by $\left(I-T_{t}\right) / t$, for all $t>0$. This is the content of Proposition 30 below. In order to take advantage of the Bellman function's properties, we suitably decompose $I-T_{t}$ into a sum of two operators which we then tame separately. At the end we pass to the limit as $t \rightarrow 0$.

Before proceeding to Proposition 30 we need a "representation formula" for symmetric contractions (see Lemma 29 below), which is a refined version of that used in [42, Theorem 3.9] for proving Proposition 3 in the case of sub-Markovian semigroups. For the purpose of stating the representation formula we introduce a bit of notation and recall a few known results concerning the linear modulus of an operator and the Gelfand transform.

Definition 27. A bounded self-adjoint operator $T$ on $L^{2}(\Omega, \nu)$ is said to be a symmetric contraction on $(\Omega, \nu)$ if for all $p \in[1, \infty]$ and $f \in L^{2}(\Omega, \nu) \cap L^{p}(\Omega, \nu)$,

$$
\|T f\|_{p} \leqslant\|f\|_{p} .
$$

We say $T$ is sub-Markovian if, in addition, $T f \geqslant 0$ whenever $f \in L^{2}(\Omega, \nu)$ and $f \geqslant 0$. A sub-Markovian operator $T$ such that $T 1=1$ is called Markovian. 
Following [11] and 29], denote by $\mathcal{P}$ the family of all finite measurable partitions of $\Omega$, partially ordered in the usual way, i.e., $\pi \leqslant \pi^{\prime}$ if and only if $\pi^{\prime}$ is a refinement of $\pi$. If $T$ is a symmetric contraction on $(\Omega, \nu), \pi=\left\{B_{1}, \ldots, B_{n}\right\}$, and $f \in L^{1}(\Omega, \nu)$, set

$$
\mathbf{T}_{\pi} f=\sum_{j=1}^{n}\left|T\left(f \chi_{B_{j}}\right)\right| .
$$

Next result was proven in [11] and [50, Lemma 3.4], see also [29, Theorems 4.1.2, 4.1.3].

Lemma 28. Suppose that $T$ is a symmetric contraction on $(\Omega, \nu)$. Then there exists a unique sub-Markovian operator $\mathbf{T}$ on $(\Omega, \nu)$ with the following properties:

(a) the operator norms of $T$ and $\mathbf{T}$ on $L^{1}(\Omega, \nu)$ are equal;

(b) $|T f| \leqslant \mathbf{T}|f|$ whenever $f \in L^{r}(\Omega, \nu)$ and $1 \leqslant r \leqslant \infty$.

When $f \in L^{1}(\Omega, \nu)$ and $f \geqslant 0$, one has that

(c) $\mathbf{T} f=\sup \left\{|T g|: g \in L^{1}(\Omega, \nu),|g| \leqslant f\right\}$;

(d) $\mathbf{T} f=\lim _{\pi \in \mathcal{P}} \mathbf{T}_{\pi} f$ in $L^{1}(\Omega, \nu)$.

The operator $\mathbf{T}$ is called linear modulus of $T$.

Gelfand theory. Let us, for the reader's convenience, briefly summarize a few basic facts about the Gelfand theory we will need in the continuation. They are taken from [49, Chapter 1] and [44, Chapter 11].

Suppose that $\nu(\Omega)<\infty$. Denote by $\widehat{\Omega}$ the maximal ideal space of the commutative unital $C^{*}$-algebra $L^{\infty}(\Omega, \nu)$. The Gelfand isomorphism $\mathcal{F}: L^{\infty}(\Omega, \nu) \rightarrow C(\widehat{\Omega})$ is given by $(\mathcal{F} f)(x)=x(f)$. We have the identities $\mathcal{F}(f g)=\mathcal{F} f \cdot \mathcal{F} g, \overline{\mathcal{F} f}=\mathcal{F} \bar{f}$ and $\mathcal{F}\left(|f|^{r}\right)=$ $|\mathcal{F} f|^{r}$ for all $r \in[1, \infty)$. We will often write $\mathcal{F} f=\hat{f}$ and $\mathcal{F}^{-1} \varphi=\check{\varphi}$. Since $\widehat{\Omega}$ is a compact Hausdorff space, by the Riesz representation theorem, the measure $\nu$ is transported to a positive Radon measure $\hat{\nu}$ on $\widehat{\Omega}$ such that

$$
\int_{\Omega} f \mathrm{~d} \nu=\int_{\widehat{\Omega}} \hat{f} \mathrm{~d} \hat{\nu}
$$

for all $f \in L^{\infty}(\Omega, \nu)$. Moreover, every $f \in L^{\infty}(\widehat{\Omega}, \hat{\nu})$ has a representative in $C(\widehat{\Omega})$, so that $L^{\infty}(\widehat{\Omega}, \hat{\nu})$ and $C(\widehat{\Omega})$ coincide as Banach spaces. It follows that, if $T$ is a symmetric contraction on $(\Omega, \nu)$, with $\nu(\Omega)<\infty$, then $\mathcal{F T F}^{-1}$ extends to a symmetric contraction on $(\widehat{\Omega}, \hat{\nu})$ which leaves $C(\widehat{\Omega})$ invariant.

If $f$ and $g$ belong to $L^{\infty}(\Omega, \nu)$, then we denote by $\widehat{f} \otimes \widehat{g}$ the continuous function on $\widehat{\Omega} \times \widehat{\Omega}$ mapping $(x, y) \mapsto \widehat{f}(x) \widehat{g}(y)$. We denote by $E$ the subspace of $C(\widehat{\Omega} \times \widehat{\Omega})$ comprising all real functions of the form $h=\sum_{j=1}^{n} \widehat{f}_{j} \otimes \widehat{g}_{j}$. If $h \in E$ and $y \in \widehat{\Omega}$, then we set $h_{y}=h(\cdot, y)$. We say that a complex measure $\mu$ on $\widehat{\Omega} \times \widehat{\Omega}$ is symmetric if $\mathrm{d} \mu(y, x)=\mathrm{d} \bar{\mu}(x, y)$.

Lemma 29. Suppose that $\nu(\Omega)<\infty$. Then for every symmetric contraction $T$ on $(\Omega, \nu)$ there exists a complex symmetric Radon measure $m_{T}$ on $\widehat{\Omega} \times \widehat{\Omega}$ with the following properties:

(i) for all $f, g \in L^{\infty}(\Omega, \nu)$,

$$
\langle T f, g\rangle=\int_{\widehat{\Omega} \times \widehat{\Omega}} \hat{f} \otimes \overline{\hat{g}} \mathrm{~d} m_{T}
$$


(ii) if $\mathbf{T}$ denotes the linear modulus of $T$, then $m_{\mathbf{T}}$ coincides with the total variation of $m_{T}$. In particular, for all $f, g \in L^{\infty}(\Omega, \nu)$,

$$
\langle\mathbf{T} f, g\rangle=\int_{\widehat{\Omega} \times \widehat{\Omega}} \hat{f} \otimes \overline{\hat{g}} \mathrm{~d}\left|m_{T}\right| .
$$

Proof. Part (i) can be proven by appropriately modifying the proof of [22, Lemma 1.4.1] (see also [42, pp. 90-91]). We omit the details.

Now let us turn towards (ii). By Lemma 28 (b) and the identity $|\mathcal{F} \varphi|=\mathcal{F}|\varphi|$, for all $\varphi \in C(\widehat{\Omega})$ and every $x \in \widehat{\Omega}$ one has that

$$
\left|\left(\mathcal{F T F}^{-1} \varphi\right)(x)\right| \leqslant\left(\mathcal{F} \mathbf{T} \mathcal{F}^{-1}|\varphi|\right)(x) .
$$

Let $h=\sum_{j=1}^{n} \widehat{f}_{j} \otimes \widehat{g}_{j} \in E$. Suppose first that $h \geqslant 0$. Then it follows from item (i), (31) and (32) that

$$
\begin{aligned}
\left|\int_{\widehat{\Omega} \times \widehat{\Omega}} h \mathrm{~d} m_{T}\right| & =\left|\int_{\widehat{\Omega}} \sum_{j=1}^{n}\left(\mathcal{F} T \mathcal{F}^{-1} \widehat{f}_{j}\right)(x) \widehat{g}_{j}(x) \mathrm{d} \hat{\nu}(x)\right| \\
& =\left|\int_{\widehat{\Omega}}\left(\mathcal{F} T \mathcal{F}^{-1} h_{x}\right)(x) \mathrm{d} \hat{\nu}(x)\right| \\
& \leqslant \int_{\widehat{\Omega}} \sum_{j=1}^{n}\left(\mathcal{F} \mathbf{T} \mathcal{F}^{-1} h_{x}\right)(x) \mathrm{d} \hat{\nu}(x) \\
& =\int_{\widehat{\Omega} \times \widehat{\Omega}} h \mathrm{~d} m_{\mathbf{T}} .
\end{aligned}
$$

Suppose now that $h$ is real-valued, but not necessarily of constant sign. Following [42, p. 91], consider a sequence of polynomials $P_{n}$ such that $P_{n}(t) \geqslant 0$ for all $t \in$ $[-1,1]$ and $P_{n}(t) \rightarrow|t|$ uniformly for $t \in[-1,1]$. In particular, for all $n \in \mathbb{N}$ we have $P_{n}\left(h /\|h\|_{\infty}\right) \geqslant 0$. Therefore, by (33),

$$
\begin{aligned}
\frac{1}{\|h\|_{\infty}}\left|\int_{\widehat{\Omega} \times \widehat{\Omega}}\right| h\left|\mathrm{~d} m_{T}\right| & =\lim _{n \rightarrow \infty}\left|\int_{\widehat{\Omega} \times \widehat{\Omega}} P_{n}\left(h /\|h\|_{\infty}\right) \mathrm{d} m_{T}\right| \\
& \leqslant \lim _{n \rightarrow \infty} \int_{\widehat{\Omega} \times \widehat{\Omega}} P_{n}\left(h /\|h\|_{\infty}\right) \mathrm{d} m_{\mathbf{T}} \\
& =\frac{1}{\|h\|_{\infty}} \int_{\widehat{\Omega} \times \widehat{\Omega}}|h| \mathrm{d} m_{\mathbf{T}} .
\end{aligned}
$$

Since $E$ is dense in $C(\widehat{\Omega} \times \widehat{\Omega} ; \mathbb{R})$, we obtain

$$
\left|m_{T}\right| \leqslant m_{\mathbf{T}}
$$

Fix $f, g \in L^{\infty}(\Omega, \nu), f, g \geqslant 0$, and $\varepsilon>0$. According to Lemma 28 (d) , there exists a partition $\pi=\left\{B_{1}, \ldots, B_{n}\right\}$ of $\Omega$ such that

$$
\langle\mathbf{T} f, g\rangle \leqslant \sum_{j=1}^{n} \int_{\Omega}\left|T\left(\chi_{B_{j}} f\right)\right| g \mathrm{~d} \nu+\varepsilon .
$$


From item (i) and the fact that $\mathcal{F}$ is an isomorphism of algebras we get

$$
\begin{aligned}
\int_{\Omega} \sum_{j=1}^{n}\left|T\left(\chi_{B_{j}} f\right)\right| g \mathrm{~d} \nu & =\int_{\Omega} \sum_{j=1}^{n} T\left(\chi_{B_{j}} f\right) \frac{\overline{T\left(\chi_{B_{j}} f\right)}}{\left|T\left(\chi_{B_{j}} f\right)\right|} g \mathrm{~d} \nu \\
& =\int_{\widehat{\Omega} \times \widehat{\Omega}} \sum_{j=1}^{n} \mathcal{F}\left(\chi_{B_{j}}\right)(x) \mathcal{F}(f)(x) \mathcal{F}\left(\frac{\overline{T\left(\chi_{B_{j}} f\right)}}{\left|T\left(\chi_{B_{j}} f\right)\right|} g\right)(y) \mathrm{d} m_{T}(x, y) \\
& \leqslant \int_{\widehat{\Omega} \times \widehat{\Omega}} \mathcal{F}\left(\sum_{j=1}^{n} \chi_{B_{j}}\right)(x) \mathcal{F}(f)(x) \mathcal{F}(g)(y) \mathrm{d}\left|m_{T}\right|(x, y) \\
& =\int_{\widehat{\Omega} \times \widehat{\Omega}} \hat{f} \otimes \hat{g} \mathrm{~d}\left|m_{T}\right| .
\end{aligned}
$$

It follows that for all nonnegative $f, g \in L^{\infty}(\Omega, \nu)$,

$$
\int_{\widehat{\Omega} \times \widehat{\Omega}} \hat{f} \otimes \hat{g} \mathrm{~d} m_{\mathbf{T}}=\langle\mathbf{T} f, g\rangle \leqslant \int_{\widehat{\Omega} \times \widehat{\Omega}} \hat{f} \otimes \hat{g} \mathrm{~d}\left|m_{T}\right| .
$$

By combining (34) with (35), we obtain

$$
\int_{\widehat{\Omega} \times \widehat{\Omega}} h \mathrm{~d} m_{\mathbf{T}}=\int_{\widehat{\Omega} \times \widehat{\Omega}} h \mathrm{~d}\left|m_{T}\right|
$$

for all $h \in E$, and the density of $E$ in $C(\widehat{\Omega} \times \widehat{\Omega} ; \mathbb{R})$ implies item (ii).

The symmetry of $m_{T}$ follows from the operator $T$ being self-adjoint.

Proposition 30. Let $p \in(2, \infty)$ and $\varepsilon \in(0,1 / 2)$. Define $\delta$ and $Q$ as in (20) and (21) respectively. Suppose that $T$ is a symmetric contraction on a $\sigma$-finite measure space $(\Omega, \nu)$. Then, for every $\phi \in\left[-\phi_{p_{\varepsilon}}, \phi_{p_{\varepsilon}}\right], f \in L^{p}(\Omega, \nu)$ and $g \in L^{q}(\Omega, \nu)$,

$$
\begin{aligned}
2 \delta \cos \phi & \left|\int_{\Omega}(I-T)(f) \bar{g} \mathrm{~d} \nu\right| \\
& \leqslant 2 \Re \int_{\Omega}\left[e^{i \phi}\left(\partial_{\zeta} Q\right)(f, g)(I-T)(f)+e^{-i \phi}\left(\partial_{\eta} Q\right)(f, g)(I-T)(g)\right] \mathrm{d} \nu .
\end{aligned}
$$

Proof. First we show that it suffices to prove the proposition in the case when $f, g$ are bounded and supported in sets of finite measure. Indeed, suppose that $f \in L^{p}(\Omega, \nu)$ and $g \in L^{q}(\Omega, \nu)$ and consider sequences $\left(s_{n}\right)$ and $\left(t_{n}\right)$ of simple functions supported in sets of finite measure, converging a.e. to $f$ and $g$, respectively, and such that $\left|s_{n}\right| \leqslant$ $|f|,\left|t_{n}\right| \leqslant|g|$. By Proposition 14. $\left|\left(\partial_{\zeta} Q\right)\left(s_{n}, t_{n}\right)\right| \lesssim \max \left\{|f|^{p-1},|g|\right\} \in L^{q}(\Omega, \nu)$ and $\left|\left(\partial_{\eta} Q\right)\left(s_{n}, t_{n}\right)\right| \lesssim|g|^{q-1} \in L^{p}(\Omega, \nu)$. Consequently, assuming that the proposition holds for all pairs $s_{n}, t_{n}$, the dominated convergence theorem and Lemma 28 (b) show that it then also holds for $f, g$.

As in [42, proof of Theorem 3.9], we may further assume that $\nu(\Omega)<\infty$. Indeed, if this is not the case, then we can replace $\Omega$ with $\Omega_{0}=(\operatorname{supp} f) \cup(\operatorname{supp} g)$ and $T$ with $\chi_{\Omega_{0}} T \chi_{\Omega_{0}}$, which is a symmetric contraction on $\left(\Omega_{0}, \nu\right)$.

Therefore, from now on we assume that $\nu(\Omega)<\infty$ and $f, g \in L^{\infty}(\Omega, \nu)$.

Recall that $\mathbf{T}$ denotes the linear modulus of $T$. We begin by splitting

$$
2 \Re \int_{\Omega}\left[e^{i \phi}\left(\partial_{\zeta} Q\right)(f, g)(I-T)(f)+e^{-i \phi}\left(\partial_{\eta} Q\right)(f, g)(I-T)(g)\right] \mathrm{d} \nu=I_{1}+I_{2},
$$


where

$$
\begin{aligned}
& I_{1}=\int_{\Omega}(1-\mathbf{T}(1)) 2 \Re\left[e^{i \phi}\left(\partial_{\zeta} Q\right)(f, g) f+e^{-i \phi}\left(\partial_{\eta} Q\right)(f, g) g\right] \mathrm{d} \nu, \\
& I_{2}=\int_{\Omega} 2 \Re\left[e^{i \phi}\left(\partial_{\zeta} Q\right)(f, g)(\mathbf{T}(1) I-T) f+e^{-i \phi}\left(\partial_{\eta} Q\right)(f, g)(\mathbf{T}(1) I-T) g\right] \mathrm{d} \nu .
\end{aligned}
$$

We first estimate $I_{1}$. An easy computation shows that

$$
2\left(\partial_{\zeta} Q\right)(f, g) f \geqslant p|f|^{p} \quad \text { and } \quad 2\left(\partial_{\eta} Q\right)(f, g) g \geqslant q|g|^{q} .
$$

Since $1-\mathbf{T}(1) \geqslant 0$ and $p|f|^{p}+q|g|^{q} \geqslant p^{1 / p} q^{1 / q}|f||g| \geqslant 2 \delta|f||g|$, it follows that

$$
I_{1} \geqslant \int_{\Omega}(1-\mathbf{T}(1)) \cos \phi\left(p|f|^{p}+q|g|^{q}\right) \mathrm{d} \nu \geqslant 2 \delta \cos \phi\left|\int_{\Omega}(1-\mathbf{T}(1)) f \bar{g} \mathrm{~d} \nu\right| .
$$

We now estimate $I_{2}$. Write

$$
I_{2}=\Re\left(e^{i \phi} K_{1}+e^{-i \phi} K_{2}\right)
$$

where

$$
K_{1}=2 \int_{\Omega}[\mathbf{T}(1) f-T f]\left(\partial_{\zeta} Q\right)(f, g) \mathrm{d} \nu \quad \text { and } \quad K_{2}=2 \int_{\Omega}[\mathbf{T}(1) g-T g]\left(\partial_{\eta} Q\right)(f, g) \mathrm{d} \nu .
$$

Let $m_{T}$ and $m_{\mathbf{T}}$ be the two measures given by Lemma 29. By the polar decomposition, there exists a measurable function $k: \widehat{\Omega} \times \widehat{\Omega} \rightarrow \mathbb{C}$ such that $|k(x, y)|=1$ for all $x, y \in \widehat{\Omega}$ and $\mathrm{d} m_{T}=k \mathrm{~d}\left|m_{T}\right|=k \mathrm{~d} m_{\mathbf{T}}$. From the definition of $Q$ and the properties of the Gelfand isomorphism $\mathcal{F}$, it follows that $\mathcal{F}\left[\left(\partial_{\zeta} Q\right)(f, g)\right]=\left(\partial_{\zeta} Q\right)(\hat{f}, \hat{g})$. Hence Lemma 29 implies that

$$
K_{1}=2 \int_{\widehat{\Omega} \times \widehat{\Omega}}(\hat{f}(y)-\hat{f}(x) k(x, y))\left(\partial_{\zeta} Q\right)(\hat{f}(y), \hat{g}(y)) \mathrm{d}\left|m_{T}\right|(x, y) .
$$

Since $m_{T}$ is symmetric, $\left|m_{T}\right|$ is symmetric and $k(y, x)=\overline{k(x, y)}$. Consequently,

$$
\begin{aligned}
K_{1}= & \int_{\widehat{\Omega} \times \widehat{\Omega}}(\hat{f}(y)-\hat{f}(x) k(x, y))\left(\partial_{\zeta} Q\right)(\hat{f}(y), \hat{g}(y)) \mathrm{d}\left|m_{T}\right|(x, y) \\
& +\int_{\widehat{\Omega} \times \widehat{\Omega}}(\hat{f}(x)-\hat{f}(y) \overline{k(x, y)})\left(\partial_{\zeta} Q\right)(\hat{f}(x), \hat{g}(x)) \mathrm{d}\left|m_{T}\right|(x, y) .
\end{aligned}
$$

It follows from the very definition of $Q$ that for every $w \in \mathbb{C}$ with $|w|=1$,

$$
\bar{w}\left(\partial_{\zeta} Q\right)(\zeta, \eta)=\left(\partial_{\zeta} Q\right)(w \zeta, \eta)=\left(\partial_{\zeta} Q\right)(w \zeta, w \eta)
$$

Since $|k(x, y)|=1$, we can continue with

$$
\begin{aligned}
K_{1}=\int_{\widehat{\Omega} \times \widehat{\Omega}} & {\left[\left(\partial_{\zeta} Q\right)(\hat{f}(y), \hat{g}(y))-\left(\partial_{\zeta} Q\right)(k(x, y) \hat{f}(x), k(x, y) \hat{g}(x))\right] } \\
\times & {[\hat{f}(y)-\hat{f}(x) k(x, y)] \mathrm{d}\left|m_{T}\right|(x, y) . }
\end{aligned}
$$

In the same way we derive the analogue of the identity above for $K_{2}$ :

$$
\begin{aligned}
K_{2}=\int_{\widehat{\Omega} \times \widehat{\Omega}} & {\left[\left(\partial_{\eta} Q\right)(\hat{f}(y), \hat{g}(y))-\left(\partial_{\eta} Q\right)(k(x, y) \hat{f}(x), k(x, y) \hat{g}(x))\right] } \\
& \times[\hat{g}(y)-\hat{g}(x) k(x, y)] \mathrm{d}\left|m_{T}\right|(x, y) .
\end{aligned}
$$


At this point we use (37) and apply Corollary 18 with

$$
\begin{aligned}
\alpha & =\hat{f}(y)-\hat{f}(x) k(x, y) & \zeta & =\hat{f}(x) k(x, y) \\
\beta & =\hat{g}(y)-\hat{g}(x) k(x, y) & \eta & =\hat{g}(x) k(x, y) .
\end{aligned}
$$

It follows that

$$
\begin{aligned}
\frac{2 I_{2}}{2 \delta \cos \phi} & \geqslant \int_{\widehat{\Omega} \times \widehat{\Omega}}|\hat{f}(y)-\hat{f}(x) k(x, y)||\hat{g}(y)-\hat{g}(x) k(x, y)| \mathrm{d}\left|m_{T}\right|(x, y) \\
& \geqslant\left|\int_{\widehat{\Omega} \times \widehat{\Omega}}(\hat{f}(y)-\hat{f}(x) k(x, y))(\overline{\hat{g}(y)}-\overline{\hat{g}(x) k(x, y)}) \mathrm{d}\right| m_{T}|(x, y)| \\
& =2\left|\int_{\widehat{\Omega} \times \widehat{\Omega}} \hat{f}(y) \overline{\hat{g}(y)} \mathrm{d}\right| m_{T}\left|(x, y)-\int_{\widehat{\Omega} \times \widehat{\Omega}} \hat{f}(x) \overline{\hat{g}(y)} \mathrm{d} m_{T}(x, y)\right| \\
& =2\left|\int_{\Omega} \mathbf{T}(1) f \bar{g} \mathrm{~d} \nu-\int_{\Omega} T(f) \bar{g} \mathrm{~d} \nu\right|
\end{aligned}
$$

We proved that

$$
I_{2} \geqslant 2 \delta \cos \phi\left|\int_{\Omega}(\mathbf{T}(1) I-T)(f) \bar{g} \mathrm{~d} \nu\right| .
$$

The proposition now follows by combining (36) with (38).

Remark 31. Observe that in the preceding proof the crucial property of $Q$, summarized in Theorem 15, was applied through Corollary 18, and was needed in order to estimate the term $I_{2}$. That term was also where the Gelfand transform and Lemma 29 were applied.

Remark 32. Suppose that $\nu(\Omega)<\infty$ and that $T$ is Markovian. Then $\mathbf{T}=T$, the measure $m_{T}$ is positive and symmetric and Lemma 29 gives

$$
\int_{\Omega}(I-T)(u) v \mathrm{~d} \nu=\int_{\widehat{\Omega} \times \widehat{\Omega}}\left(\int_{\{x, y\}} \mathcal{G} \hat{u} \cdot \hat{v} \mathrm{~d} \nu_{x, y}\right) \mathrm{d} m_{T}(x, y),
$$

for all $u, v \in L^{\infty}(\Omega, \nu)$. Therefore, in this case, Proposition 30 immediately follows from Proposition 26 ,

As discussed at the beginning of this section, Theorem 9 (bilinear embedding) will follow once we confirm the next corollary.

Corollary 33. Let $p \in(2, \infty)$ and $\varepsilon \in(0,1 / 2)$. Define $\delta$ and $Q$ as in (20) and (21), respectively. For all $f \in \mathrm{D}\left(\mathcal{A}_{p}\right), g \in \mathrm{D}\left(\mathcal{A}_{q}\right)$ and every $\phi \in\left[-\phi_{p_{\varepsilon}}, \phi_{p_{\varepsilon}}\right]$, inequality (16) holds with $B_{0}=2 \delta \cos \phi$.

Proof. Since $\left(T_{t}\right)_{t>0}$ is a symmetric contraction semigroup and, by Proposition 14, $\left(\partial_{\zeta} Q\right)(f, g) \in L^{q}(\Omega, \nu)$ and $\left(\partial_{\eta} Q\right)(f, g) \in L^{p}(\Omega, \nu)$, the corollary follows from applying Proposition 30 with $T_{t}$ in place of $T$, dividing by $t$ both sides of the ensuing inequality and passing to the limit as $t \rightarrow 0$.

Remark 34. In order to apply the heat-flow argument of Section 4 we used Proposition 3. However, Corollary 33 immediately gives a different proof of this result. Indeed, by Corollary 33 applied with $g=0$,

$$
\Re \int_{\Omega} e^{i \phi}\left(\partial_{\zeta} Q\right)(f, 0) \mathcal{A} f \mathrm{~d} \nu \geqslant 0
$$


for all $f \in \mathrm{D}\left(\mathcal{A}_{p}\right)$ and $\phi \in\left[-\phi_{p_{\varepsilon}}, \phi_{p_{\varepsilon}}\right]$. Since $\left(\partial_{\zeta} Q\right)(\zeta, 0)=(p+2 \delta) \bar{\zeta}|\zeta|^{p-2}$ for any $\zeta \in \mathbb{C}$, and $\phi_{p_{\varepsilon}} \rightarrow \phi_{p}$ as $\varepsilon \rightarrow 0$, the inequality above can be rewritten as the sectorial inequality

$$
\left.\left.\left|\Im \int_{\Omega} \mathcal{A}_{p} f \cdot \bar{f}\right| f\right|^{p-2} \mathrm{~d} \nu\left|\leqslant \cot \phi_{p} \cdot \Re \int_{\Omega} \mathcal{A}_{p} f \cdot \bar{f}\right| f\right|^{p-2} \mathrm{~d} \nu,
$$

which is well known to be equivalent to Proposition 3 .

Acknowledgements. The first author was partially supported by the Gruppo Nazionale per l'Analisi Matematica, la Probabilità e le loro Applicazioni (GNAMPA) of the Istituto Nazionale di Alta Matematica (INdAM). The second author was partially supported by the Ministry of Higher Education, Science and Technology of Slovenia (research program Analysis and Geometry, contract no. P1-0291). A part of this paper was written during the first author's visit at Faculty of Mathematics and Physics, University of Ljubljana, and during the second author's visit at Dipartimento di matematica, University of Genova. The authors wish to thank the said institutions for their kind hospitality.

\section{REFERENCES}

[1] J. F. ANkeR: $L^{p}$ Fourier multipliers on Riemannian symmetric spaces of the noncompact type, Ann. of Math. (2) 132 (1990), no. 3, 597-628.

[2] J. F. Anker, N. LohouÉ: Multiplicateurs sur certains espaces symétriques, Amer. J. Math. 108 (1986), no. 6, 1303-1353.

[3] D. BAKRY: Sur l'interpolation complexe des semi-groupes de diffusion, Séminaire de Probabilités XXIII, Lecture Notes in Math., Springer, 1372 (1989), 1-20.

[4] J. Bennett: Heat-flow monotonicity related to some inequalities in Euclidean analysis, Proceedings of the 8th International Conference on Harmonic Analysis and Partial Differential Equations, El Escorial, Spain, Contemp. Math. 505 (2010), Amer. Math. Soc., Providence, RI, pp. 85-96.

[5] J. Bennett, A. Carbery, M. Christ, T. TaO: The Brascamp-Lieb Inequalities: Finiteness, Structure and Extremals, Geom. and Funct. Anal. 17 (2008), 1343-1415.

[6] D. L. Burkholder: Boundary value problems and sharp inequalities for martingale transforms, Ann. Prob. 12, no. 3 (1984), 647-702.

[7] D. L. Burkholder: A proof of Petczyński's conjecture for the Haar system, Studia Math. 91 (1988), no. 1, 79-83.

[8] D. L. Burkholder: Explorations in martingale theory and its applications, Ecole d'Eté de Probabilités de Saint-Flour XIX - 1989, Lecture Notes in Math. 1464, Springer, Berlin (1991), 1-66.

[9] A. Carbonaro: Functional calculus for some perturbations of the Ornstein-Uhlenbeck operator, Math. Z. 262 (2009), no. 2, 313-347.

[10] A. Carbonaro, O. DragiČević: Bellman function and dimension-free estimates in a theorem of Bakry, J. Funct. Anal. 265 (2013), 1085-1104.

[11] R. V. Chacon, U. Krengel: Linear modulus of a linear operator, Proc. Amer. Math. Soc. 15 (1964), 553-559.

[12] J. L. Clerc, E. M. Stein: $L^{p}$-multipliers for noncompact symmetric spaces, Proc. Nat. Acad. Sci. U.S.A. 71 (1974), 3911-3912.

[13] M. Cowling: Harmonic analysis on semigroups, Ann. Math. (2) 117 (1983), 267-283.

[14] M. Cowling, I. Doust, A. McIntosh, A. Yagi: Banach space operators with a bounded $H^{\infty}$ functional calculus, J. Austral. Math. Soc. 60 (1996), 51-89.

[15] M. Cowling, S. Giulini, A. Hulanicki, G. Mauceri: Spectral multipliers for a distinguished Laplacian on certain groups of exponential growth, Studia Math. 111 no. 2 (1994), 103-121.

[16] M. Cowling, S. Meda: Harmonic analysis and ultracontractivity, Trans. Amer. Math. Soc. 340 (1993), 733-752.

[17] O. Dragičević, S. Treil, A. Volberg: A theorem about three quadratic forms, Int. Math. Res. Not. IMRN (2008), Art. ID rnn 072.

[18] O. DragičEvić, A. VolBerg: Bellman functions and dimensionless estimates of Littlewood-Paley type, J. Oper. Theory 56 (2006), no. 1, 167-198. 
[19] O. Dragičević, A. Volberg: Linear dimension-free estimates in the embedding theorem for Schrödinger operators, J. London Math. Soc. (2) 85 (2012), 191-222.

[20] O. DragičEviĆ, A. Volberg: Bilinear embedding for real elliptic differential operators in divergence form with potentials, J. Funct. Anal. 261 no. 10 (2011), 2816-2828.

[21] T. X. Duong, E. M. Ouhabaz, A. Sikora: Plancherel-type estimates and sharp spectral multipliers, J. Funct. Anal. 196 no. 2 (2002), 443-485.

[22] M. Fukushima: Dirichlet Forms and Markov Processes, North-Holland and Kodansha, 1980.

[23] J. García-Cuerva, G. Mauceri, S. Meda, P. Sjögren, J. L. Torrea: Functional Calculus for the Ornstein-Uhlenbeck Operator, J. Funct. Anal. 183 (2001), 413-450.

[24] G. Garrigós, A. Seeger: Characterizations of Hankel multipliers, Math. Ann. 342 (2008), no. $1,31-68$.

[25] W. Hebisch, G. Mauceri, S. Meda: Holomorphy of spectral multipliers of the OrnsteinUhlenbeck operator, J. Funct. Anal. 210 (2004), no. 1, 101-124.

[26] Y. Heo, F. Nazarov, A. Seeger: Radial Fourier multipliers in high dimensions, Acta Math. 206 (2011), 55-92.

[27] L. Hörmander: Estimates for translation invariant operators in $L^{p}$ spaces, Acta Math. 104, no. 1-2 (1960), 93-140.

[28] N. J. Kalton, L. Weis: The $H^{\infty}$-calculus and sums of closed operators, Math. Ann. 321 no. 2 (2001), 319-345.

[29] U. Krengel: Ergodic theorems, Walter de Gruyter and Co., Berlin, 1985.

[30] C. KRIEGLER: Analyticity angle for non-commutative diffusion semigroups, J. London Math. Soc. (2) 83 (2011), no. 1, 168-186.

[31] P. C. Kunstmann, Ž. Štrkalu: $H^{\infty}$-calculus for submarkovian generators, Proc. Amer. Math. Soc. 131, no. 7 (2003), 2081-2088.

[32] V. A. Liskevich, M. A. Perelmuter: Analyticity of submarkovian generators, Proc. Amer. Math. Soc. 123, no. 4 (1995), 1097-1104.

[33] G. Mauceri, S. MedA, P. Sjögren: Sharp estimates for the Ornstein-Uhlenbeck operator, Ann. Sc. Norm. Super. Pisa Cl. Sci. (5) 3 (2004), no. 3, 447-480.

[34] G. Mauceri, S. Meda, M. Vallarino: Estimates for functions of the Laplacian on manifolds with bounded geometry, Math. Res. Lett. 16 (2009), no. 5, 861-879.

[35] S. MedA: A general multiplier theorem, Proc. Amer. Math. Soc. 110 (1990), 639-647.

[36] S. G. Mihlin: On the multipliers of Fourier integrals (Russian), Dokl. Akad. Nauk SSSR (N. S.), 109 (1956), 701-703.

[37] S. G. Minlin: Fourier integrals and multiple singular integrals (Russian), Vestnik Leningrad. Univ. Set. Mat. Mech. Astr. 12 (1957), no. 7, 143-155.

[38] F. NAzArov, S. Treil: The Hunt for a Bellman function: applications to estimates of singular integral operators and to other classical problems in harmonic analysis, St. Petersburg Math. J. 8 (1997), no. 5, 721-824.

[39] F. Nazarov, S. Treil, A. Volberg: The Bellman functions and two-weight inequalities for Haar multipliers, J. Amer. Math. Soc. 12 (1999), no. 4, 909-928.

[40] F. Nazarov, S. Treil, A. Volberg: Bellman function in stochastic control and harmonic analysis (how our Bellman function got its name), Oper. Theory: Advances Appl. 129 (2001), 393-423, Birkhäuser Verlag.

[41] F. Nazarov, A. Volberg: Heat extension of the Beurling operator and estimates for its norm, St. Petersburg Math. J. 15 (2004), no. 4, 563-573.

[42] E. M. Ouhabaz: Analysis of Heat Equations on Domains, London Mathematical Society Monograph Series, vol. 31, Princeton and Oxford, 2005.

[43] S. Petermichl, A. VolberG: Heating of the Beurling operator: weakly quasiregular maps on the plane are quasiregular, Duke Math. J. 112 (2002), no. 2, 281-305.

[44] W. Rudin: Functional Analysis, Second edition, McGraw-Hill, New York, 1991.

[45] E. SASSO: Functional calculus for the Laguerre operator, Math. Z. 249 (2005), no. 3, 683-711.

[46] R. J. Stanton, P. A. Tomas: Expansions for spherical functions on noncompact symmetric spaces, Acta Math. 140 (1978) no. 3-4, 251-276.

[47] E. M. Stein: Topics in Harmonic Analysis Related to the Littlewood-Paley Theory, Princeton University Press, Princeton, New Jersey (1970).

[48] R. Strichartz: Analysis of the Laplacian on the complete Riemannian manifold, J. Funct. Anal. 52 (1983), 48-79. 
[49] M. TAKesaki: Theory of operator algebras. I, Encyclopaedia of Mathematical Sciences, 124. Operator Algebras and Non-commutative Geometry, 5. Springer-Verlag, Berlin, 2002.

[50] R. J. Taggart: Pointwise convergence for semigroups in vector-valued $L^{p}$ spaces, Math. Z. 261 (2009), no. 4, 933-949.

[51] M. E. TAYLOR: $L^{p}$-estimates on functions of the laplace operator, Duke Math. J. 58 (1989), no. 3, 973-993.

[52] M. E. TAYLOR: Functions of the Laplace operator on manifolds with lower Ricci and injectivity bounds, Comm. Partial Differential Equations 34 (2009), no. 7-9, 1114-1126.

[53] A. VolberG: Bellman approach to some problems in harmonic analysis, Séminaire équations aux dérivées partielles (2001-2002), Exposé n XIX, Ecole Polytéchnique, 14 p.

[54] J. WitTwer: Survey article: a user's guide to Bellman functions, Rocky Mountain J. Math. 41, no. 3 (2011), 631-661.

Andrea Carbonaro, University of Birmingham, School of Mathematics, Watson Building, Edgbaston, Birmingham B15 2TT, United Kingdom

Current address: Università degli Studi di Genova, Dipartimento di Matematica, Via Dodecaneso, 3516146 Genova, Italy

E-mail address: carbonaro@dima.unige.it

Oliver Dragičević, University of Ljubljana, Faculty of Mathematics and Physics, JadRANSKa 21, SI-1000 Ljubluana, Slovenia

E-mail address: oliver.dragicevic@fmf.uni-lj.si 